\title{
Inhomogeneous molecular ring around the $B[e]$ supergiant LHA 120-S 73 ${ }^{\star}, \star \star$
}

\author{
M. Kraus ${ }^{1,2}$, L. S. Cidale ${ }^{3,4}$, M. L. Arias ${ }^{3,4}$, G. Maravelias ${ }^{1}$, D. H. Nickeler ${ }^{1}$, A. F. Torres ${ }^{3,4}$, M. Borges Fernandes ${ }^{5}$, \\ A. Aret $^{2}$, M. Curé 6 , R. Vallverdú ${ }^{3,4}$, and R. H. Barbá ${ }^{7}$
}

\author{
1 Astronomický ústav, Akademie věd České republiky, Fričova 298, 25165 Ondřejov, Czech Republic \\ e-mail: michaela.kraus@asu.cas.cz \\ 2 Tartu Observatory, Tõravere, 61602 Tartumaa, Estonia \\ 3 Departamento de Espectroscopía Estelar, Facultad de Ciencias Astronómicas y Geofísicas, \\ Universidad Nacional de La Plata (UNLP), Paseo del Bosque s/n, 1900 La Plata, Argentina \\ ${ }^{4}$ Instituto de Astrofísica de La Plata, CCT La Plata, CONICET-UNLP, Paseo del Bosque s/n, 1900, La Plata, Argentina \\ 5 Observatório Nacional, Rua General José Cristino 77, 20921-400 São Cristovão, Rio de Janeiro, Brazil \\ 6 Instituto de Física y Astronomía, Facultad de Ciencias, Universidad de Valparaíso, Av. Gran Bretaña 1111, Casilla 5030, Valparaíso, \\ Chile \\ 7 Departamento de Física y Astronomía, Universidad de La Serena, Cisternas 1200 Norte, La Serena, Chile
}

Received 11 March 2016 / Accepted 22 June 2016

\begin{abstract}
Context. B[e] supergiants are evolved massive stars, enshrouded in a dense wind and surrounded by a molecular and dusty disk. The mechanisms that drive phases of enhanced mass loss and mass ejections, responsible for the shaping of the circumstellar material of these objects, are still unclear.

Aims. We aim to improve our knowledge on the structure and dynamics of the circumstellar disk of the Large Magellanic Cloud B[e] supergiant LHA $120-\mathrm{S} 73$.

Methods. High-resolution optical and near-infrared spectroscopic data were obtained over a period of 16 and 7 yr, respectively. The spectra cover the diagnostic emission lines from [Ca II] and [O I], as well as the $\mathrm{CO}$ bands. These features trace the disk at different distances from the star. We analyzed the kinematics of the individual emission regions by modeling their emission profiles. A lowresolution mid-infrared spectrum was obtained as well, which provides information on the composition of the dusty disk.

Results. All diagnostic emission features display double-peaked line profiles, which we interpret as due to Keplerian rotation. We find that the profile of each forbidden line contains contributions from two spatially clearly distinct rings. In total, we find that LHA 120-S 73 is surrounded by at least four individual rings of material with alternating densities (or by a disk with strongly nonmonotonic radial density distribution). Moreover, we find that the molecular ring must have gaps or at least strong density inhomogeneities, or in other words, a clumpy structure. The optical spectra additionally display a broad emission feature at $6160-6180 \AA$, which we interpret as molecular emission from TiO. The mid-infrared spectrum displays features of oxygen- and carbon-rich grain species, which indicates a long-lived, stable dusty disk.

We cannot confirm the previously reported high value for the stellar rotation velocity. He I $\lambda 5876$ is the only clearly detectable pure atmospheric absorption line in our data. Its line profile is strongly variable in both width and shape and resembles of those seen in non-radially pulsating stars. A proper determination of the real underlying stellar rotation velocity is hence not possible.

Conclusions. The existence of multiple stable and clumpy rings of alternating density recalls ring structures around planets. Although there is currently insufficient observational evidence, it is tempting to propose a scenario with one (or more) minor bodies or planets revolving around LHA 120-S 73 and stabilizing the ring system, in analogy to the shepherd moons in planetary systems.
\end{abstract}

Key words. circumstellar matter - stars: early-type - supergiants - stars: individual: LHA 120-S 73 - infrared: stars

\footnotetext{
^ Based on observations: (1) with the 1.52-m and 2.2-m telescopes at the European Southern Observatory (La Silla, Chile), under the programme 076.D-0609(A) and under the agreement with the Observatório Nacional-MCT (Brazil); (2) at the Gemini Observatory, which is operated by the Association of Universities for Research in Astronomy, Inc., under a cooperative agreement with the NSF on behalf of the Gemini partnership: the National Science Foundation (United States), the Science and Technology Facilities Council (United Kingdom), the National Research Council (Canada), CONICYT (Chile), the Australian Research Council (Australia), Ministério da Ciência, Tecnologia e Inovação (Brazil) and Ministerio de Ciencia, Tecnología e Innovación Productiva (Argentina), under the programmes GS-2004B-Q-54, GS-2010B-Q-31, and GS-2012B-Q-90; (3) at Complejo Astronómico El Leoncito operated under agreement between the Consejo Nacional de Investigaciones Científicas y Técnicas de la República Argentina and the National Universities of La Plata, Córdoba and San Juan (Visiting Astronomer: A.F.T.); and (4) with the du Pont Telescope at Las Campanas Observatory, Chile, under the programme CNTAC 2008-02 (Visiting Astronomer: R.B. and A.F.T.).

$\star \star$ Presented spectroscopic data are only available at the CDS via anonymous ftp to cdsarc.u-strasbg.fr (130.79.128.5) or via http://cdsarc.u-strasbg.fr/viz-bin/qcat?J/A+A/593/A112
} 


\section{Introduction}

$\mathrm{B}[\mathrm{e}]$ supergiants (B[e]SGs) are evolved massive stars (Zickgraf et al. 1986). They form a subgroup of objects displaying the $\mathrm{B}[\mathrm{e}]$ phenomenon (Lamers et al. 1998). Their optical spectra display a hybrid character, meaning that they present broad and intense Balmer emission lines and simultaneously narrow emission lines of low-excitation permitted and forbidden transitions of low-ionized and neutral elements (i.e., Fe II, [Fe II] and [O I]). In addition, the stars exhibit a very strong near- or mid-infrared excess.

The $\mathrm{B}[\mathrm{e}]$ phenomenon is typically related to the physical conditions of the gaseous and dusty shells, rings, or disks surrounding a hot star and not to the properties of the star itself. The origin of circumstellar envelopes of $\mathrm{B}$ [e]SGs is attributed to the mass lost from the star either through dense, aspherical stellar winds or through sudden mass ejections expected to occur during short-lived phases in the post-main-sequence evolution of the stars. The possible structure of the B[e]SG circumstellar envelopes was described by Zickgraf et al. (1985), who proposed an empirical model, consisting of a hot and fast line-driven wind in the polar regions, and a slow, much cooler and denser (by a factor of $10^{2}-10^{3}$ ) wind in the equatorial region.

In this cool and dense equatorial region, the material condenses into molecules and dust. Molecular emission from carbon monoxide $(\mathrm{CO})$, tracing the inner rim of the molecular region, was found in many B[e]SGs (McGregor et al. 1988a,b, 1989; Morris et al. 1996; Liermann et al. 2010; Cidale et al. 2012; Oksala et al. 2012, 2013; Wheelwright et al. 2012b; Kraus et al. 2013b). Emission from less prominent molecules such as titanium oxide (TiO, Zickgraf et al. 1989; Torres et al. 2012) and silicon oxide ( $\mathrm{SiO}$, Kraus et al. 2015b) was also reported for a few objects. In the outer parts of these equatorial regions, where the temperature drops below the dust condensation value, dust forms. The large amount of dust connected with $\mathrm{B}$ [e]SGs is visible as strong near- and mid-infrared excess emission (Zickgraf et al. 1986; Bonanos et al. 2009, 2010) and resolved spectral dust features (Kastner et al. 2006, 2010). Interferometric observations of some Galactic $\mathrm{B}[\mathrm{e}] \mathrm{SG}$ s revealed that the dust is concentrated in a ring (Domiciano de Souza et al. 2007, 2011; Cidale et al. 2012). In a few cases, the central object turned out to be a close binary, and the dusty rings are circumbinary (Millour et al. 2011; Wheelwright et al. 2012a; Wang et al. 2012).

Investigations of the kinematics within the gaseous (atomic and molecular) disk regions often revealed that it is consistent with Keplerian rotation (Millour et al. 2011; Aret et al. 2012; Cidale et al. 2012; Wheelwright et al. 2012b; Kraus et al. 2010, 2013b, 2014, 2015b; Muratore et al. 2015). In some cases, observations support evidence of disk variability as seen in LHA 115S 18 (Torres et al. 2012) and HD 327083 (Kraus et al. 2013a), sudden disk formation as in LHA 115-S 65 (Oksala et al. 2012), and disk dissipation as in CI Cam (Liermann et al. 2014).

To improve our knowledge on the disk formation process, the physical properties of the disks, and the timescales of possible variability and its origin, extensive observational monitoring of individual objects is indispensable. In the present work, we focus on the Large Magellanic Cloud (LMC) B[e]SG star LHA 120-S 73. We investigate its gaseous (atomic and molecular) environment based on multi-epoch high-resolution optical and near-infrared spectroscopic observations and its dusty disk based on low-resolution mid-infrared data.

\section{Star}

LHA 120-S 73: (HD 268 835, RMC 66, IRAS 04571-6954, HIP 22 989, $\alpha=04^{\mathrm{h}} 56^{\mathrm{m}} 47$ s.077 and $\delta=-69^{\circ} 50^{\prime} 24^{\prime \prime} .79$; J2000) is a bright emission-line star in the LMC (Henize 1956). It has previously been classified by Feast et al. (1960) as a peculiar A-type emission-line star. Fehrenbach et al. (1969) reported on numerous forbidden emission lines of singly ionized iron. Strong infrared excess emission was found by Stahl et al. (1983). These authors also classified the object as a massive late B-type supergiant, surrounded by a dense envelope, which mimics an equivalent spectral type of an early A-type star, and a dusty shell. Stahl \& Wolf (1986) discovered narrow nebular emission lines, such as [OI] and [N II] lines. As one of the dusty peculiar emission-line stars in the Magellanic Clouds, it was recognized as a B[e]SG by Zickgraf et al. (1986), who introduced this designation.

The stellar parameters derived by Stahl et al. (1983) are $T_{\text {eff }}=12000 \mathrm{~K}, M_{\text {bol }}=-8.94 \mathrm{mag}, L=3 \times 10^{5} L_{\odot}, R=125 R_{\odot}$, $M=30 M_{\odot}$, and $E(B-V)=0.12$. Similar values were derived by van Genderen et al. (1983): $T_{\mathrm{eff}}=12000 \mathrm{~K}, M_{\mathrm{bol}}=$ $-9.4 \mathrm{mag}, L=4.4 \times 10^{5} L_{\odot}, R=143 R_{\odot}, M=37 M_{\odot}$, and $E(B-V)=0.26$. The wind terminal velocity was determined to $v_{\infty} \simeq 300 \mathrm{~km} \mathrm{~s}^{-1}$ based on the observed low-resolution UV absorption spectrum, and estimates for the mass-loss rate range from $\dot{M}=6 \times 10^{-6} M_{\odot} \mathrm{yr}^{-1}$ (Muratorio \& Friedjung 1988) to $\dot{M}=3 \times 10^{-5} M_{\odot} \mathrm{yr}^{-1}$ (Stahl et al. 1983).

Photometric observations by van Genderen \& Sterken (2002) over a period of about two years indicated slight variability. The authors identified two possible periods, a long-term period ( $\sim 380$ days) with an amplitude of $\sim 0.06 \mathrm{mag}$ superimposed on a short-term period ( $\sim 55.5$ days) with an amplitude of $\sim 0.03 \mathrm{mag}$. Based on the ASAS $V$-band light curve spanning ten years of observations, Szczygieł et al. (2010) observed a pulsation-type oscillation with a period of $\sim 224$ days and an amplitude of $\sim 0.08 \mathrm{mag}$. This period is about four times longer than the 55.5 days identified by van Genderen \& Sterken (2002). The periods were ascribed to possible pulsations, and van Genderen \& Sterken (2002) classified LHA 120-S 73 as an $\alpha$ Cyg variable. These authors mentioned that LHA 120-S 73 shares many similarities with luminous blue variables in an S Dor outburst stage. Furthermore, Stahl et al. (1983) and Stahl \& Wolf (1986) suggested that the envelope might have been ejected during an S Dor-like outburst. However, no S Dor typical variability was reported for LHA 120-S 73 so far.

The optical spectrum of LHA 120-S 73 exhibits countless narrow emission lines, which display no shift with respect to the stellar radial velocity, while the higher Balmer lines are blueshifted. The optical spectra were described in detail by Stahl et al. (1983, 1985), who also found that the Balmer lines as well as some strong Fe II and the Ca II K lines display P Cygni profiles. Only a few typically very shallow photospheric absorption lines were reported.

Near- and mid-infrared excess emission is seen in the spectral energy distribution of LHA 120-S 73 (Zickgraf et al. 1986; Bonanos et al. 2009), and observations from both Spitzer and Herschel space telescopes revealed that LHA 120-S 73 is one of nine evolved massive stars in the LMC with notable far-infrared emission (van Loon et al. 2010; Jones et al. 2015). Moreover, emission features from amorphous and christalline silicates and from polycyclic aromatic hydrocarbons (PAHs) were detected in the data obtained with the Spitzer Space Telescope Infrared Spectrograph (IRS; Kastner et al. 2010). Intrinsic polarization due to dust was observed by Magalhaes (1992), suggesting 
Table 1. Observation summary.

\begin{tabular}{|c|c|c|c|c|}
\hline $\begin{array}{l}\text { Date } \\
\text { (UT) }\end{array}$ & $\begin{array}{l}t_{\exp } \\
(\mathrm{s})\end{array}$ & $\begin{array}{l}\text { Instrument/ } \\
\text { Observatory }\end{array}$ & Resolution & $\begin{array}{l}\text { Wavelength } \\
\text { range }\end{array}$ \\
\hline 1999-12-17 & 4500 & FEROS/ESO-La Silla & 55000 & $3600-9200 \AA$ \\
\hline $2005-12-12$ & 1500 & FEROS/ESO-La Silla & 55000 & $3600-9200 \AA$ \\
\hline 2014-11-28 & 1600 & FEROS/ESO-La Silla & 55000 & $3600-9200 \AA$ \\
\hline $2015-10-12$ & 1400 & FEROS/ESO-La Silla & 55000 & $3600-9200 \AA$ \\
\hline $2008-11-13$ & 1800 & Echelle-du Pont/LCO & 45000 & $3480-10150 \AA$ \\
\hline 2006-01-16 & 2700 & REOSC/CASLEO & 13000 & $4560-7100 \AA$ \\
\hline $2012-11-26$ & 2700 & REOSC/CASLEO & 13000 & 5780-9100 ̊ \\
\hline $2012-11-27$ & 1800 & REOSC/CASLEO & 13000 & 5780-9100 ̊ \\
\hline 2004-12-17 & 1800 & Phoenix/GEMINI-South & 50000 & $2.290-2.299 \mu \mathrm{m}$ \\
\hline $2010-12-24$ & 5920 & Phoenix/GEMINI-South & 50000 & $2.290-2.299 \mu \mathrm{m}$ \\
\hline 2011-01-04 & 5920 & Phoenix/GEMINI-South & 50000 & $2.319-2.330 \mu \mathrm{m}$ \\
\hline $2012-10-03$ & 400 & T-ReCS/GEMINI-South & 100 & $8.0-13.0 \mu \mathrm{m}$ \\
\hline
\end{tabular}

a non-spherically symmetric distribution of the dust, probably confined to a disk.

A disk seen close to pole-on was also inferred by Zickgraf et al. (1986) and Muratorio \& Friedjung (1988), considering the huge amount of narrow permitted and forbidden emission lines of low-ionized metals (predominantly Fe II) coexisting with broad PCygni absorption components from a relatively high-velocity wind. Aret et al. (2012) analyzed the kinematics of the two most valuable tracers of high-density gas: the lines of [OI] and [Ca II]. They found that the disk is rotating in Keplerian fashion and is seen under an inclination angle of $28 \pm 1^{\circ}$, in agreement with a more pole-on orientation of the star.

Near-infrared spectra of the star display pronounced, apparently stable molecular emission in both ${ }^{12} \mathrm{CO}$ and ${ }^{13} \mathrm{CO}$ (McGregor et al. 1988b; Liermann et al. 2010; Oksala et al. 2013). The amount of identified ${ }^{13} \mathrm{CO}$ implies that the surface of LHA $120-S 73$ was strongly enriched in ${ }^{13} \mathrm{C}$ at the time the material was released. The CO-band emission typically originates from a (rotating) molecular gas ring, however, in these past observations the spectral resolution was too low to resolve the kinematics of the $\mathrm{CO}$ emitting region.

\section{Observations}

Mid- and near-infrared as well as optical spectra of LHA 120S 73 were obtained. A summary of all observations is presented in Table 1, where we list the observing date (Col. 1), exposure time (Col. 2), instrument and observatory (Col. 3), spectral resolution (Col. 4), and wavelength coverage (Col. 5).

\subsection{Mid-infrared spectrum}

A low-resolution $(R \sim 100)$ mid-infrared spectrum in the $N$ band, covering the range $8-13 \mu \mathrm{m}$, was acquired in 2012 with the T-ReCS spectrograph attached to the 8-m telescope at GEMINI-South (Chile). The spectrum was processed using standard IRAF ${ }^{1}$ tasks for mid-infrared spectroscopic data reduction to obtain stacked images for the sky and difference frames,

\footnotetext{
1 IRAF is distributed by the National Optical Astronomy Observatories, which are operated by the Association of Universities for Research in Astronomy, Inc., under cooperative agreement with the National Science Foundation.
}

wavelength calibration, and to extract the target and standard telluric star spectra.

Telluric correction of the target spectrum was performed using standard IRAF tasks. Finally, we obtained a rough flux calibration by multiplying the spectrum with a blackbody continuum of the same temperature as the telluric standard star. The flux scale was thereby determined from the available mid-infrared photometry of the science target.

\subsection{Near-infrared spectra}

We obtained high-resolution $(R \sim 50000)$ long-slit $K$-band spectra in December 2004, December 2010, and January 2011 with the visitor spectrograph Phoenix mounted at GEMINI-South (Chile). The spectra were obtained using two different filters, K4396 and K4308, covering a spectral range of 2.290-2.299 $\mu \mathrm{m}$ and $2.319-2.330 \mu \mathrm{m}$, respectively. This wavelength range was chosen because it covers the positions of the first and second band head of the rotation-vibrational transitions of the $\mathrm{CO}$ firstovertone bands.

A late-type B main-sequence telluric standard star was observed close in time to the target and at similar airmass. For ideal sky subtraction, the observations were taken in an ABBA nod pattern. Data reduction and telluric correction was performed using standard IRAF tasks.

The final, normalized, and continuum-subtracted nearinfrared spectra are shown in Fig. 1. The data taken in 2004 are noisy, but the structure of the CO-band head is still recognizable.

\subsection{Optical spectra}

Five medium-resolution spectra were obtained between 2006 and 2012 using the REOSC echelle spectrograph attached to the Jorge Sahade 2.15-m telescope at Complejo Astronómico El Leoncito (CASLEO, Argentina). REOSC provides a resolving power of $R \sim 13000$. The data from 2006 cover the spectral range 4560-7100 $\AA$ and were taken with grating 580 with $4001 \mathrm{~mm}^{-1}$. Those from 2012 cover the spectral range 5780-9100 $\AA$ and were observed with grating 180 with $3161 \mathrm{~mm}^{-1}$. Data reduction was performed using standard IRAF tasks. In 2012, two spectra were taken each night, which were co-added for a better signal-to-noise ratio $(\mathrm{S} / \mathrm{N})$.

In addition, four high-resolution spectra were obtained between 1999 and 2015 with the Fiber-fed Extended Range Optical 

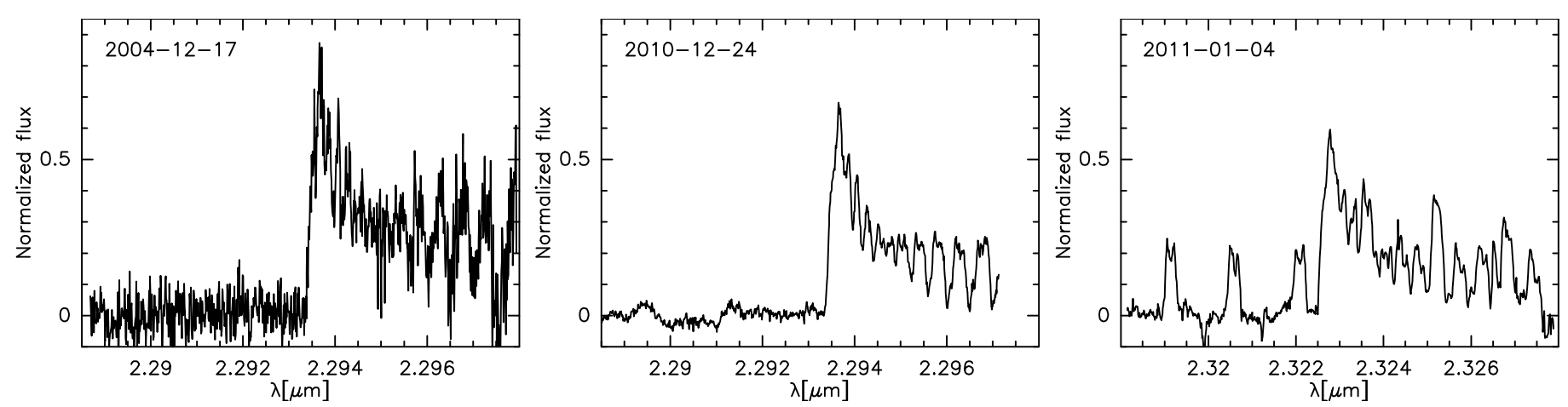

Fig. 1. Normalized and continuum-subtracted near-infrared Phoenix spectra of LHA 120-S 73. Observations taken in 2004 (left) and 2010 (middle) cover the first CO-band head, those taken in 2011 (right) cover the second.

Spectrograph (FEROS Kaufer et al. 1999). During the observations in December 1999, the spectrograph was attached to the 1.52-m telescope, while all other observations were carried out with the spectrograph attached to the 2.2-m telescope, both at the European Southern Observatory in La Silla (Chile). FEROS is a bench-mounted echelle spectrograph with fibers, which cover a sky area of $2^{\prime \prime}$ of diameter, with a wavelength coverage from $3600 \AA$ to $9200 \AA$ and a spectral resolution of $R=55000$ (in the region around $6000 \AA$ ). The data were reduced using the FEROS reduction pipeline. A standard star was observed each night for telluric correction, except for the night in 1999. Telluric correction was performed using standard IRAF tasks, and for the data from 1999, a telluric template from another night was applied.

Moreover, two high-resolution spectra were acquired in 2008 with the echelle spectrograph attached to the 2.5-m $d u$ Pont telescope at Las Campanas Observatory (LCO, Chile). The wavelength coverage ranges from $3480 \AA$ to $10150 \AA$ with a resolution of $\sim 45000$. A Tek $52 \mathrm{k} \times 2 \mathrm{k}$ CCD detector with pixel size of 24 microns and a $1 \times 4^{\prime \prime}$ slit were used. The data were reduced using standard IRAF tasks. For these observations, no telluric standard star was taken, hence the correction could not be performed.

All spectra were corrected for the heliocentric velocity and for the stellar radial velocity of $261 \mathrm{~km} \mathrm{~s}^{-1}$ determined by Aret et al. (2012).

\section{Results}

\subsection{CO-band emission}

The high resolution of the near-infrared spectra unveils that the CO-band head structures display kinematical broadening. This is obvious from the fully resolved double-peaked line profiles of the three individual rotation-vibrational transitions blueward of the second band head (right panel of Fig. 1). In addition, the first band head displays a slightly broadened and asymmetric structure with a blue shoulder and a red peak. This is characteristic of the emission originating in a rotating molecular disk or ring (Carr et al. 1993; Carr 1995; Najita et al. 1996; Kraus et al. 2000). Hence, we modeled the CO bands using the code developed by Kraus et al. (2000), which is suitable to compute CO-band emission in local thermodynamic equilibrium (LTE) from a rotating disk. As was found in previous investigations of $\mathrm{CO}$ bands in $\mathrm{B}[\mathrm{e}] \mathrm{SGs}$, the molecular spectra are typically well reproduced considering solely a narrow rotating ring because the observable $\mathrm{CO}$ emission originates from the hot inner edge
Table 2. CO model parameters.

\begin{tabular}{cccc}
\hline \hline $\begin{array}{c}T_{\mathrm{CO}} \\
(\mathrm{K})\end{array}$ & $\begin{array}{c}N_{\mathrm{CO}} \\
\left(\mathrm{cm}^{-2}\right)\end{array}$ & $\begin{array}{c}v_{\text {rot }} \\
\left(\mathrm{km} \mathrm{s}^{-1}\right)\end{array}$ & $\begin{array}{c}i \\
\left(^{\circ}\right)\end{array}$ \\
\hline $2850 \pm 100$ & $(6 \pm 1) \times 10^{20}$ & $34 \pm 1$ & 28 \\
\hline
\end{tabular}

Notes. The inclination angle is taken from Aret et al. (2012) and is a fixed input value.

of the molecular disk (Kraus et al. 2000, 2013b; Kraus 2009; Liermann et al. 2010; Cidale et al. 2012; Muratore et al. 2015). In addition, the involved column densities in these rings are high, justifying the LTE assumption. This reduces the number of free model parameters to three. These are the temperature of the $\mathrm{CO}$ ring $T_{\mathrm{CO}}$, the ring column density along the line of sight $N_{\mathrm{CO}}$, and the deprojected rotation velocity of the molecular ring $v_{\text {rot }}$. For the inclination angle, we adopted the value of $28^{\circ}$ estimated by Aret et al. (2012). To compare them with the observations, the theoretical spectra were convolved with the resolution of the Phoenix spectrograph.

The quality of the 2004 spectrum of LHA $120-\mathrm{S} 73$ is poor. To determine the physical parameters of the $\mathrm{CO}$ emitting region, we thus modeled the data taken in 2010/2011, which have the advantage of a much better quality, a longer wavelength coverage (two band heads), and the spectra were taken close in time with a separation of only 11 days.

Our spectra reveal no indication for emission from the hydrogen Pfund series. This lack of Pfund lines is in agreement with former studies of Oksala et al. (2013), who found a contribution of the Pfund series in LHA 120-S 73 only for wavelengths longer than $2.329 \mu \mathrm{m}$. This red part of the $K$-band spectrum is not covered by our observations. The minimum wavelength of the Pfund line contribution found by Oksala et al. (2013) corresponds to the line $\operatorname{Pf}(34)$, and this cutoff in the Pfund series at relatively low quantum number implies a high electron density of $\sim 10^{15} \mathrm{~cm}^{-3}$ in the Pfund line emitting region (see, e.g., Kraus 2000).

The best fit to the observed, normalized, and continuumsubtracted pure $\mathrm{CO}$ band spectra is shown in Fig. 2, and the fit parameters are listed in Table 2. The upper panel of Fig. 2 shows the full computed spectral range in comparison to the observations, while the lower panels highlight the two band head regions. Obviously, there is some disagreement between the model and the observations. It is apparently impossible to fit the strengths of both band head structures with a unique set of parameters. Hence, the presented fit and its parameters provide a 

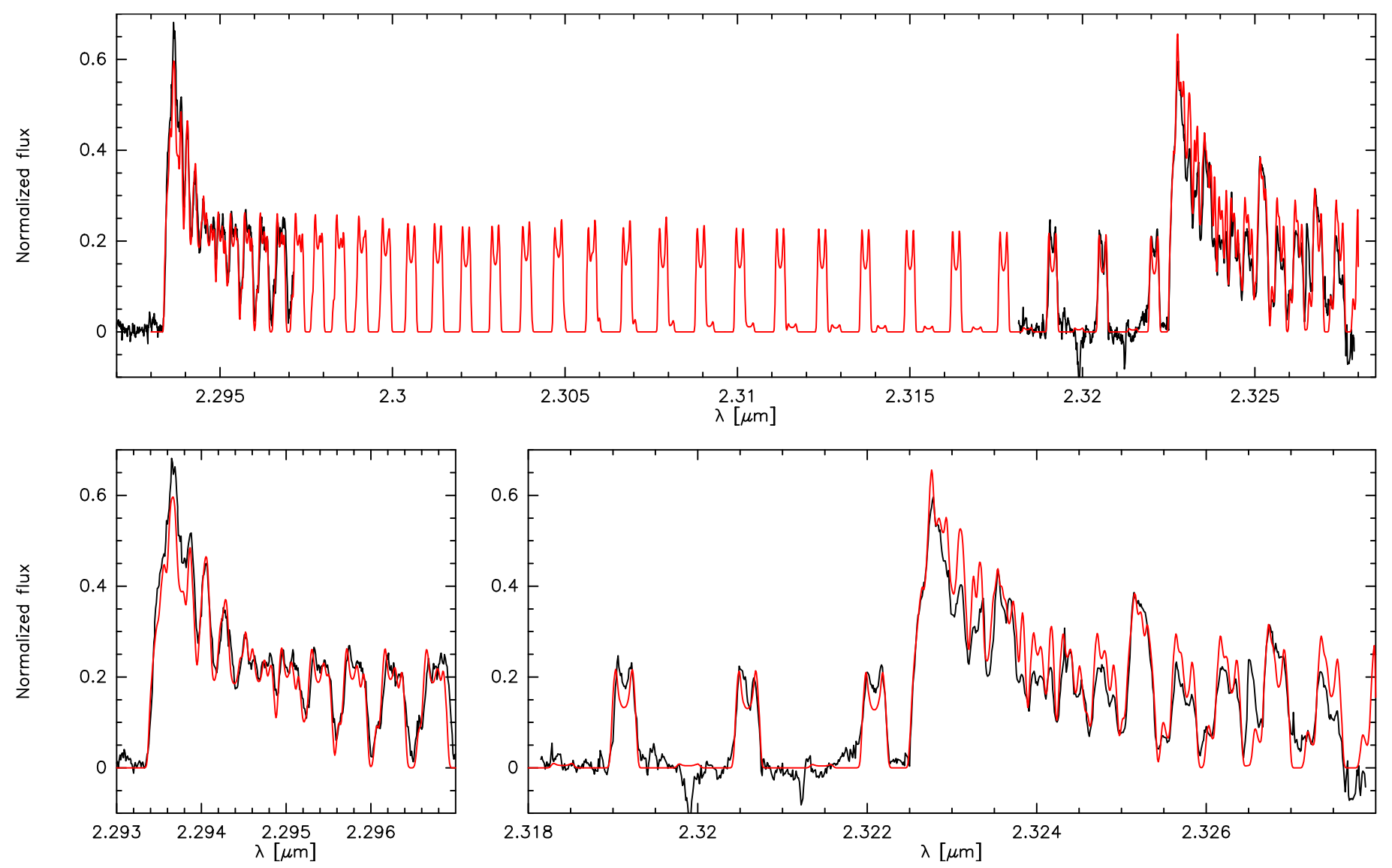

Fig. 2. Model fits (red) to the observed, normalized, and continuum-subtracted (black) first and second CO-band heads of LHA 120-S 73. The top panel shows the complete CO-band structure, the bottom panels only the observed band head regions.

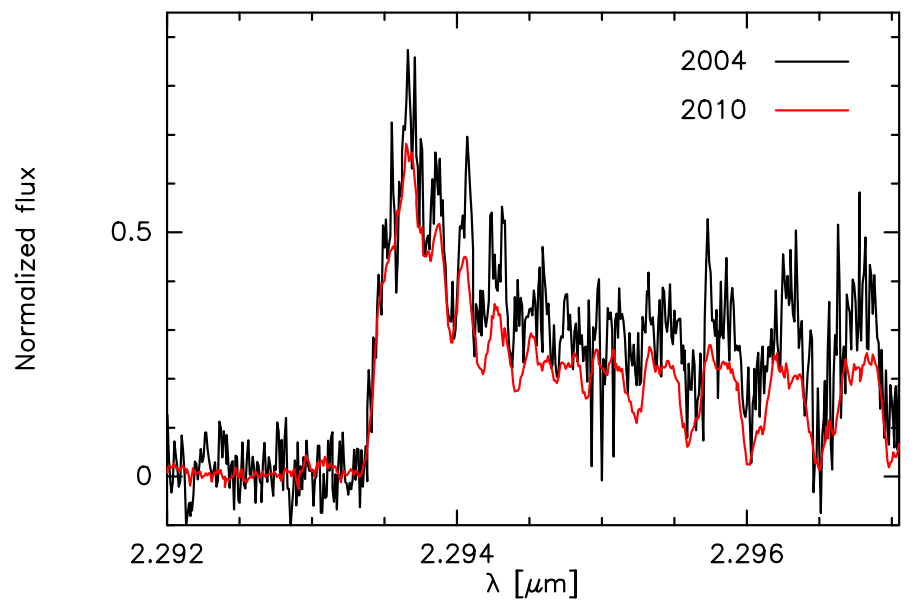

Fig. 3. Comparison of the intensities of the first CO-band head observed with Phoenix in 2004 and 2010.

compromise, so that the intensity of the first band head is not too strongly underestimated and the one of the second band head not too strongly overestimated. Such a mismatch has not been reported for any other $\mathrm{CO}$-band emission from evolved massive stars and requires some further investigation.

When we compare the first CO-band head observed in 2004 with the one observed in 2010 (see Fig. 3) we see two important facts: the intensity of the emission is definitely lower in 2010 , but the width of the band head itself remains constant. The latter implies that the rotation velocity of the $\mathrm{CO}$ gas did not change, while the former suggests a change in column density and/or size of the emitting area.

To test this hypothesis, we fixed both the $\mathrm{CO}$ temperature and rotation velocity to the best-fitting values listed in Table 2. Then we modeled the three observed spectral pieces individually, by purely modifying the column density. This procedure is possible because the column density needed to fit the observations is high, and optical depth effects become visible. This means that the shape of the band heads is determined by the column density. In a purely optically thin scenario, the intensity of the CO-band emission would simply scale with the column density and a proper determination of the column density would not be possible.

The best-fitting parameters for the three individual spectral pieces are given in Table 3, where we list the year of the observation (Col. 1), the $\mathrm{CO}$ column density value ( $\mathrm{Col} .2)$, and the fitting parameter $f$ (Col. 3, in arbitrary units). The latter parameter basically represents a normalized emission area. The larger errors in the parameters to fit the data from 2004 are due to the lower quality of the spectrum. The resulting fits are shown in Fig. 4. The fits to the individual spectra from 2010 and 2011 are considerably better than the one to the combined data set shown in Fig. 2. For comparison purpose, we list the values obtained from the fit to the combined observations (Fig. 2) in the last row of Table 3.

\subsection{Dust emission}

The mid-infrared spectrum spans from $8 \mu \mathrm{m}$ to $13 \mu \mathrm{m}$ (see Fig. 5). Its shape and intensity are comparable to the Spitzer 

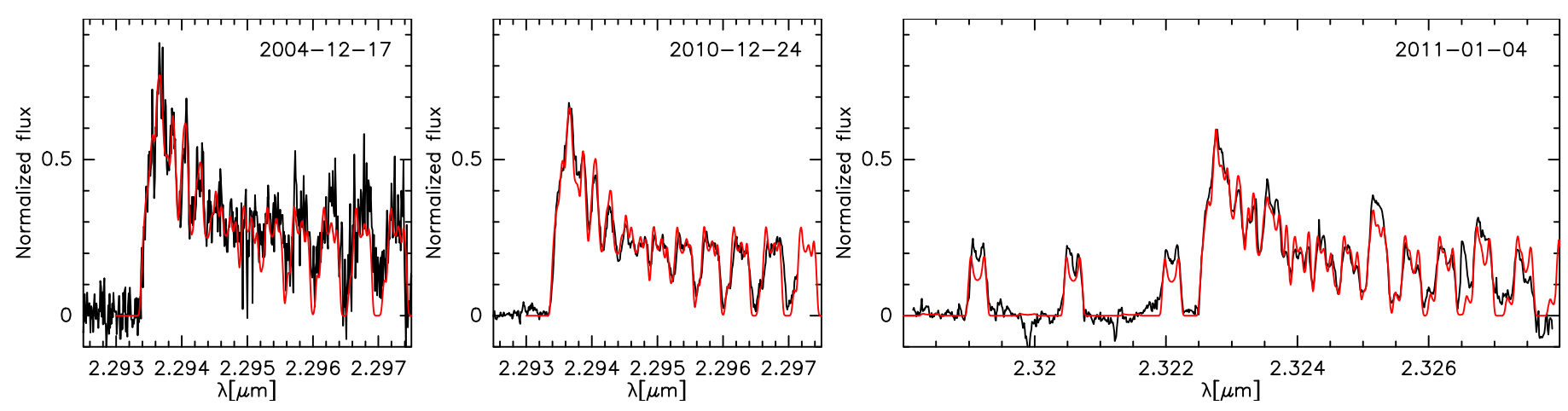

Fig. 4. Model fits (red) to the normalized and continuum-subtracted (black) observations. Each model has a different column density and emitting area.

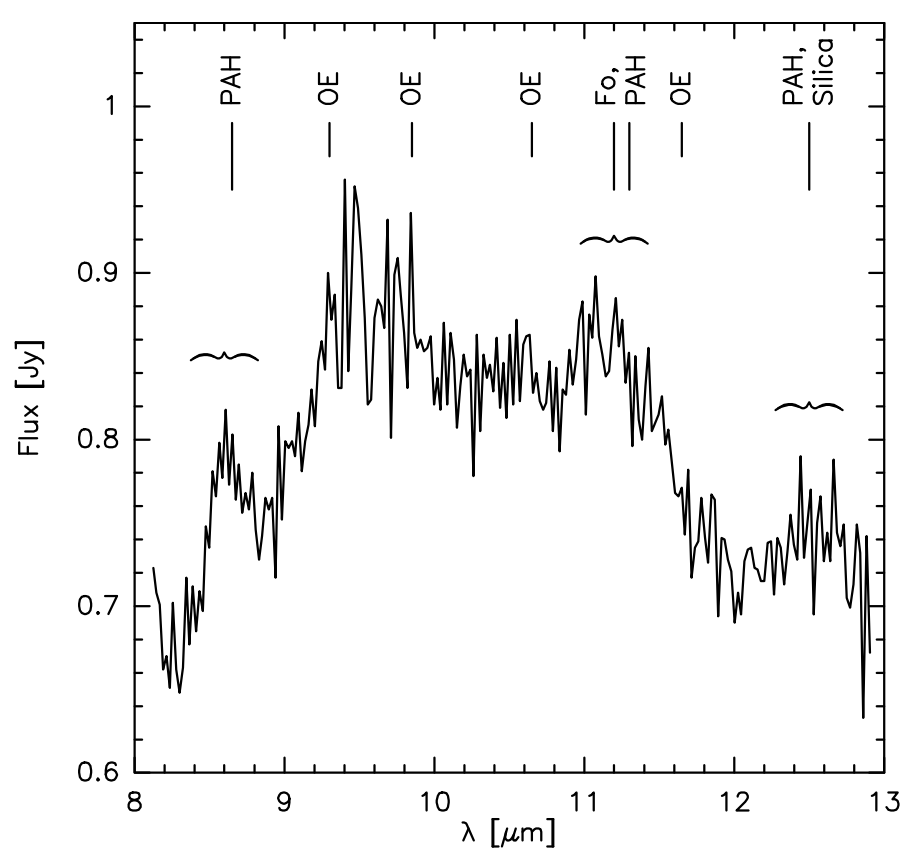

Fig. 5. Flux-calibrated mid-infrared spectrum. Prominent emission features are identified: PAH - polycyclic aromatic hydrocarbons, OE ortho enstatite, Fo - forsterite, and silica.

Table 3. Density and fitting parameters.

\begin{tabular}{ccc}
\hline \hline Observation & $\begin{array}{c}N_{\mathrm{CO}} \\
\left(10^{20} \mathrm{~cm}^{-2}\right)\end{array}$ & $\begin{array}{c}f \\
(\text { a.u. })\end{array}$ \\
\hline 2004 & $7 \pm 1$ & $3.65_{-0.11}^{+0.14}$ \\
2010 & $5 \pm 0.5$ & $3.46_{-0.10}^{+0.13}$ \\
2011 & $4 \pm 0.5$ & $3.01_{-0.12}^{+0.15}$ \\
$2010+2011$ & $6 \pm 1$ & $2.94_{-0.14}^{+0.20}$ \\
\hline
\end{tabular}

spectrum (Kastner et al. 2010). It clearly shows a prominent broad emission structure and, on top, several sharp emission features. According to the dust emission templates of van Boekel et al. (2005), we tentatively ascribe the broad structure to emission from large amorphous silicates (olivine, pyroxene, and silica), and we identify the sharp features as emission from crystalline silicates (forsterite, ortho enstatite) together with weak emission from PAHs. LHA 120-S 73 hence displays a dual-dust chemistry, in which oxygen- and carbon-based grain particles coexist.

\subsection{Optical emission features}

The optical spectra of LHA 120-S 73 are crowded with countless narrow emission lines, particularly in the red portion. Most of these lines belong to permitted and forbidden transitions of Fe II. Only a few photospheric absorption lines are seen. We refrain from repeating the description of the optical spectra because they were described in great detail by Stahl et al. (1983, 1985), Zickgraf et al. (1986), and Muratorio \& Friedjung (1988). Moreover, Zickgraf et al. (1986) reported that no indication for spectroscopic variability was seen in data taken sporadically over two decades. The same statement is true for our data. The spectral features show no significant changes over the 16-year period covered by our observations. This spectral stability can be seen in Figs. 6 and 7, where we compare the shape and strength of the Balmer lines and of different forbidden emission lines, respectively. As the CASLEO spectra have considerably lower resolution, we show here only the high-resolution (FEROS and du Pont) observations. In general, the line profiles from the different epochs agree very well. However, we observe small variations in the absorption component of the Balmer lines. In addition, the double-peaked [Ca II] $\lambda 7291$ line shows variability in the peak intensities (right panel of Fig. 7): while the profile had a more intense red peak in 1999, it displays a more intense blue peak in 2014. In the other years (2005 and 2015) the profile appears symmetric.

The [O I] $\lambda 5577$ line also seems to display double-peaked profiles, although this line is very weak and hence very noisy, which distorts the real profile shape. The [O I] $\lambda 6300$ line profiles appear single peaked at first glance, but we also note slight asymmetries. The [O I] $\lambda 6300$ line from the du Pont spectra was omitted from Fig. 7 because the shape and strength of its profile is strongly affected by telluric pollution.

\subsubsection{Kinematics of the forbidden lines}

The forbidden emission lines of [Ca II] and [O I] are of particular interest because they were suggested to originate in the high - density Keplerian disks of B[e]SGs (Kraus et al. 2007, 2010; Aret et al. 2012). Moreover, these forbidden lines were found to trace different density regimes, with the [Ca II] lines forming closest to the star, the [O I] $\lambda 5577$ line at similar or slightly larger distances, and the [O I] $\lambda 6300$ line forming even farther out. In a recent study, Aret et al. (2016) found that the profiles of the [Ca II] and [O I] lines in the two investigated Galactic B[e]SGs (3 Pup and MWC 349) can be reproduced assuming that each emission originates from an individual Keplerian ring tracing a different distance from the star. When combining the information 

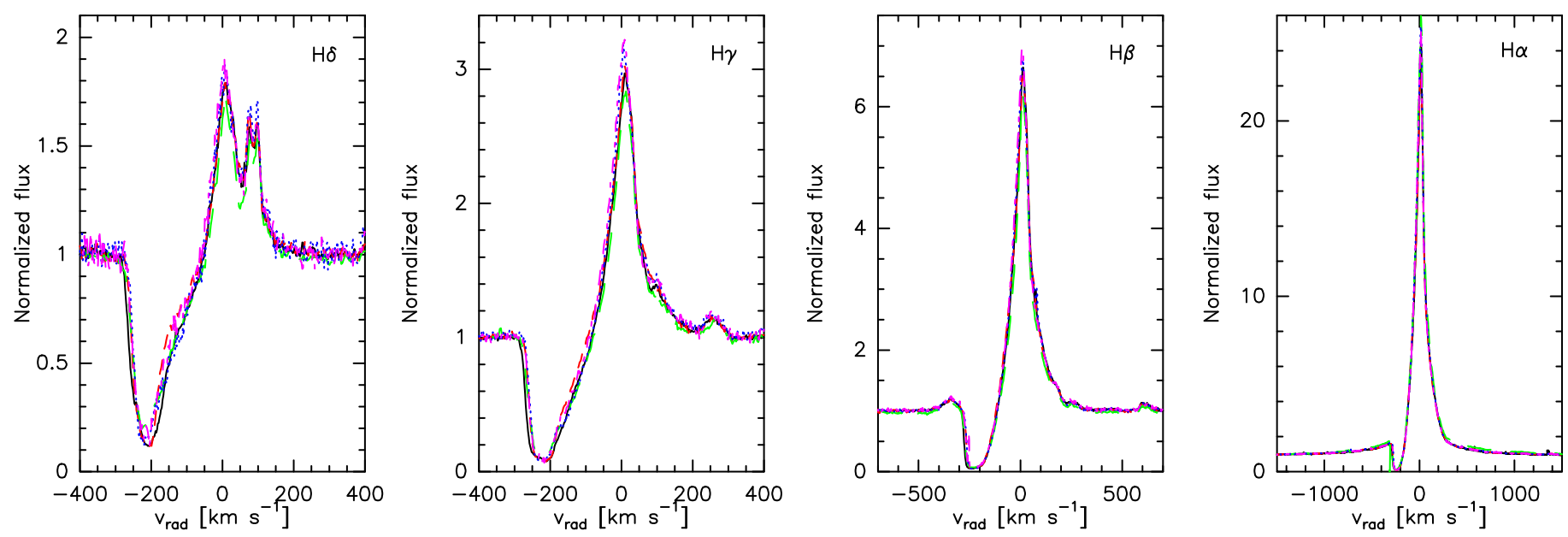

Fig. 6. Comparison of the strength and shape of the Balmer lines of LHA 120-S 73 seen in FEROS spectra from 1999 (black, solid), 2005 (red, dashed), 2014 (blue, dotted), and 2015 (purple, dashed-dotted), and in the du Pont data from 2008 (green, long-dashed).
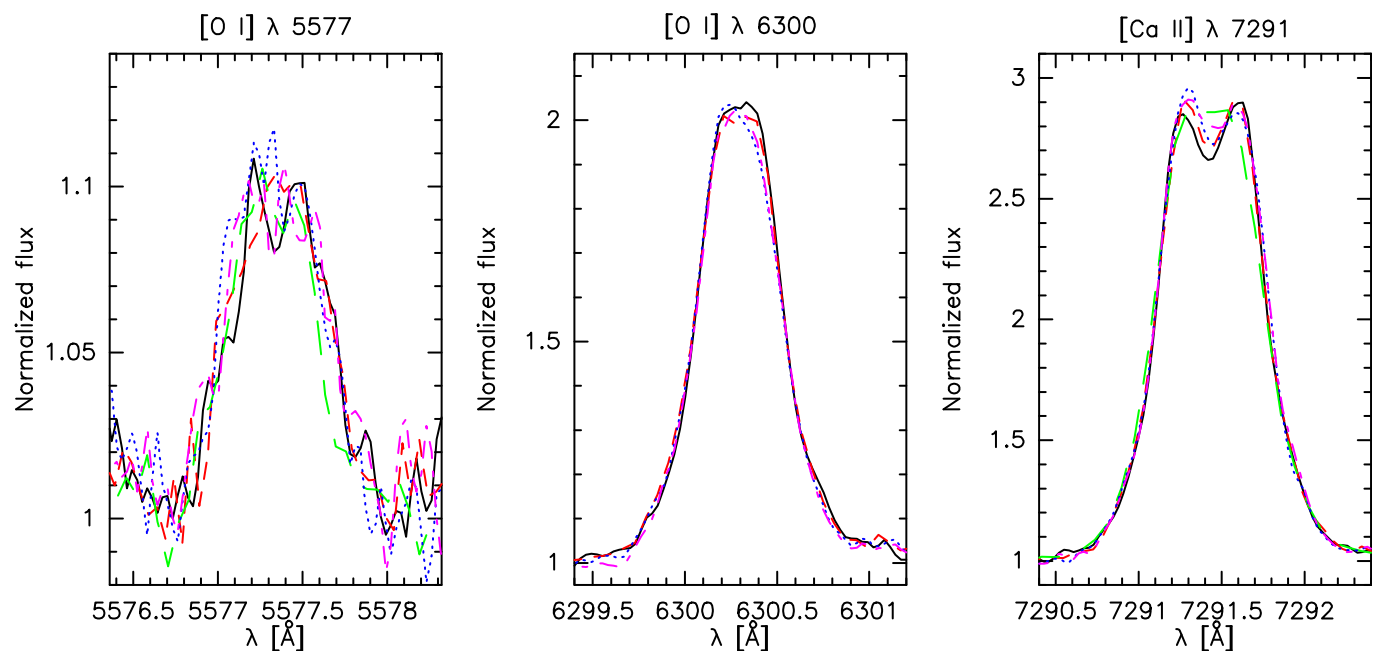

Fig. 7. Comparison of the strength and shape of some forbidden lines of LHA 120-S 73 seen in FEROS spectra from 1999 (black, solid), 2005 (red, dashed), 2014 (blue, dotted), and 2015 (purple, dashed-dotted), and in the du Pont data from 2008 (green, long-dashed), except for the [O I] $\lambda 6300$ line, which could not be corrected for telluric pollution.

from different atomic and molecular disk tracers for 3 Pup, it became clear that the disk around this star cannot be continuous but must be confined into seperate rings, at least one atomic and one molecular. This conclusion was drawn from the lack of hot CO-band head emission (Kraus 2016).

The low CO temperature of only $2800 \mathrm{~K}$ obtained from our models suggests that the molecular gas disk around LHA 120$\mathrm{S} 73$ also has a sharp inner edge traced by the $\mathrm{CO}$ bands, indicative of an abrupt change in density. If the emission of the forbidden lines is confined to a narrow gas ring, then either this ring should agree with the position of the molecular gas, or it should be clearly detached from it. To study the kinematics stored in the profiles of the forbidden emission lines in LHA 120-S 73, we followed the approach of Aret et al. (2016) and applied a purely kinematical model. We assumed that the emission is generated within a ring of gas with a rotational and a Gaussian velocity component. The latter contains a combination of at least the thermal velocity of $\sim 1-2 \mathrm{~km} \mathrm{~s}^{-1}$ and the spectral resolution of FEROS of $\sim 5.5-6.5 \mathrm{~km} \mathrm{~s}^{-1}$. An additional component due to some internal (turbulent) motion of the gas might be present as well, but will be neglected at first.
The [Ca II] $\lambda 7291$ line and the [OI] $\lambda 5577$ line display a clear double-peaked profile. For a test computation, we used the $\mathrm{CO}$ emitting region as starting point and computed the profile resulting from a ring with a rotational velocity of $34 \mathrm{~km} \mathrm{~s}^{-1}$ and a Gaussian component of $6.0 \mathrm{~km} \mathrm{~s}^{-1}$. This profile was then compared to the observed double-peaked line profiles (see the top panels of Fig. 8). Obviously, a single Keplerian ring with this rotational velocity cannot reproduce the observations, whose peaks are much narrower at the top of the lines and significantly wider in their wings. Consequently, the observed line profile must consist of at least two components, one with a higher and one with a lower rotation velocity than the $\mathrm{CO}$ gas. Therefore, we next tested the possibility of two Keplerian rings. We found that a combination of two rings can fit the observed double-peaked lines very well. The fits are shown in the bottom panels of Fig. 8 and the values needed for the individual velocity components are listed in Table 4. Interestingly, the emission from the ring at larger distance from the star displays no additional broadening beyond spectral resolution, while the emission from the inner ring requires a velocity component of $\sim 8-9 \mathrm{~km} \mathrm{~s}^{-1}$ in addition to the thermal and spectral resolution broadening. While parts 

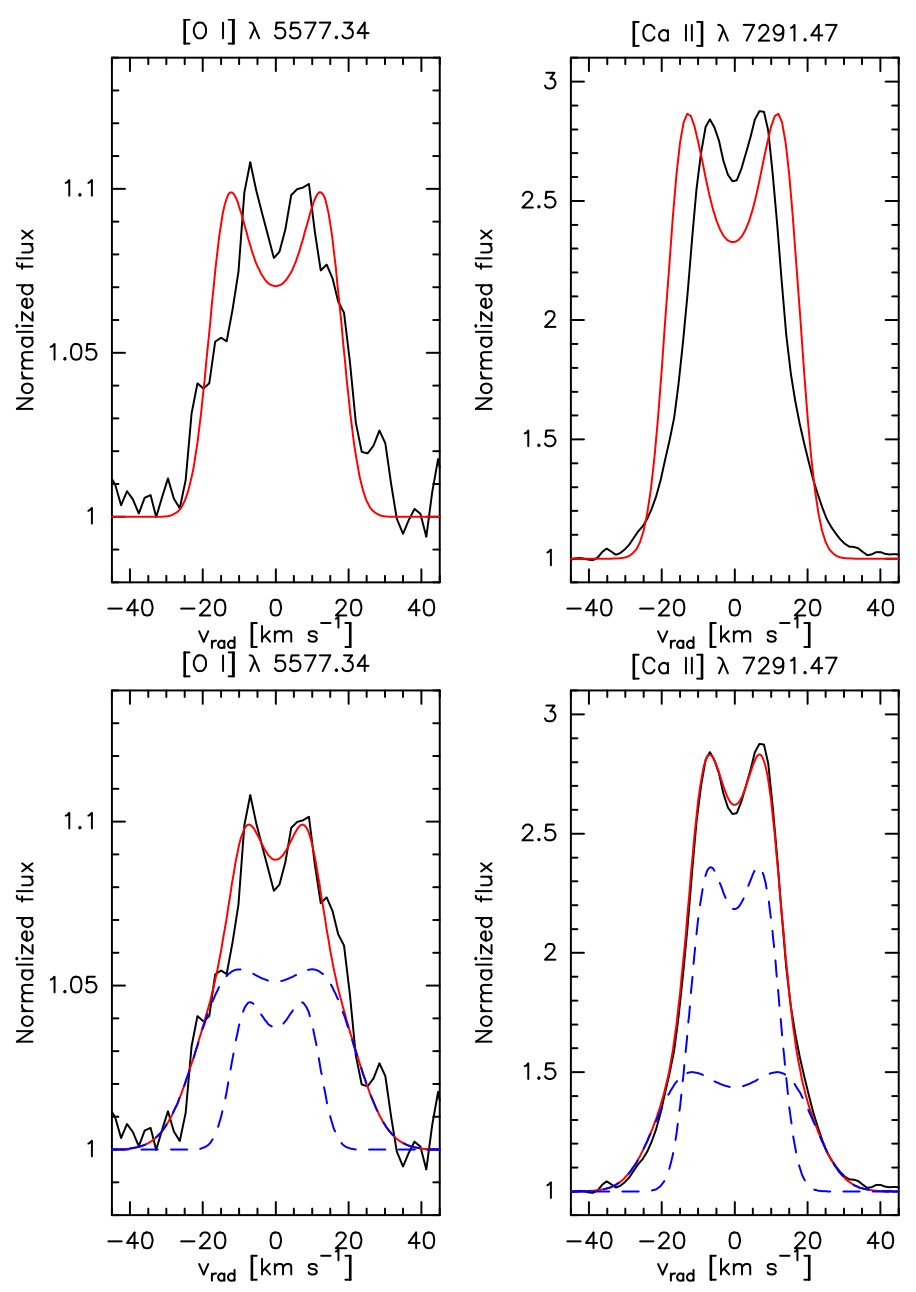

Fig. 8. Comparison of the observed line profiles seen in the FEROS spectrum from 1999 (black) with theoretical model fits (red) consisting of a single (top) and two Keplerian rings (bottom). In the latter, the blue lines show the contributions of the individual rings.

Table 4. Profile kinematics of the forbidden lines.

\begin{tabular}{lcccc}
\hline \hline Line & $\begin{array}{c}v_{\text {rot, } 1} \\
\left(\mathrm{~km} \mathrm{~s}^{-1}\right)\end{array}$ & $\begin{array}{c}v_{\text {gauss,1 }} \\
\left(\mathrm{km} \mathrm{s}^{-1}\right)\end{array}$ & $\begin{array}{c}v_{\text {rot,2 }} \\
\left(\mathrm{km} \mathrm{s}^{-1}\right)\end{array}$ & $\begin{array}{c}v_{\text {gauss,2 }} \\
\left(\mathrm{km} \mathrm{s}^{-1}\right)\end{array}$ \\
\hline$[\mathrm{Ca}$ II $] 7291$ & $39 \pm 1$ & $10 \pm 1$ & $22 \pm 1$ & $5.5 \pm 1$ \\
{$[\mathrm{O}$ I] 5577 } & $38 \pm 2$ & $11 \pm 2$ & $22 \pm 2$ & $5 \pm 2$ \\
{$[\mathrm{O}$ I $] 6300$} & $33 \pm 1$ & $11 \pm 1$ & $16 \pm 1$ & $7 \pm 1$ \\
\hline
\end{tabular}

of this velocity might be caused by intrinsic (turbulent) motion of the gas, we interpret this component as an indicator that the inner ring is not confined but has a certain radial extension.

Both double-peaked line profiles can be fit with basically identical velocity components, which agrees with earlier findings that these lines occur in regions of very similar density (e.g., Aret et al. 2012). The excitation conditions for the levels from which the [Ca II] lines emerge are non-trivial. These lines can be populated both through collisions from the ground level and through decay of energetically higher levels by the Ca II triplet line emission. This makes it difficult to analyze the level population without a proper radiation transfer model for Ca II. The situation for the OI lines is easier. The levels from which the forbidden lines emerge are decoupled from the rest of the atom so that they are purely collisionally excited. As their emission is optimized within a rather narrow density range (see, e.g.,
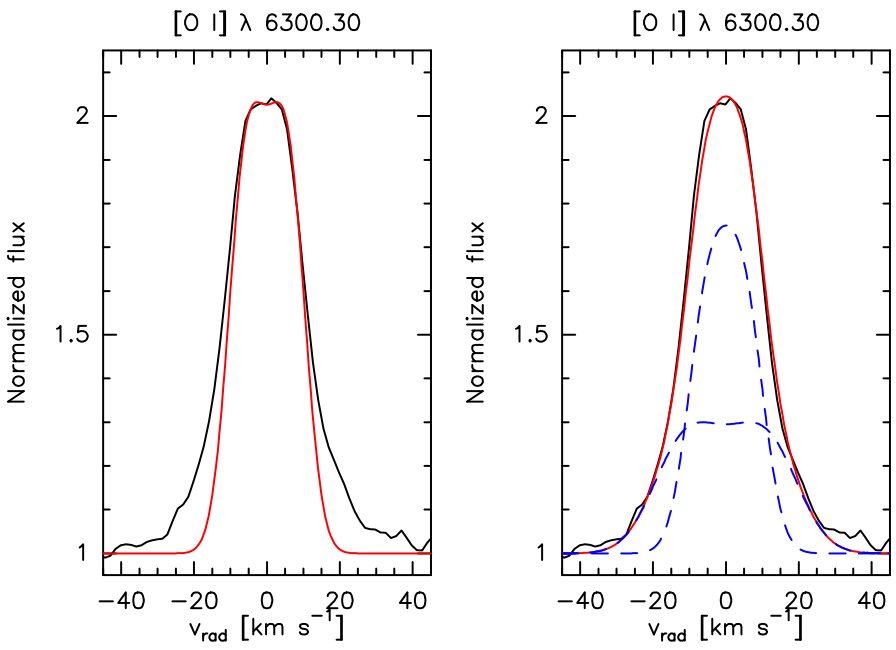

Fig. 9. Comparison of the observed line profile seen in the FEROS spectrum from 1999 (black) with theoretical model fits (red) consisting of a single (left) and two Keplerian rings (right). In the latter, the blue lines show the contributions of the individual rings.

Ercolano \& Owen 2016), the necessity of two rings suggests that the material in the environment of LHA 120 -S 73 has radial density inhomogeneities. This means that at larger distance from the star similarly high densities must be achieved as closer to the star to have a sufficiently high level population of the levels from which the forbidden lines emerge.

We now consider the [O I] $\lambda 6300$ line, which appears singlepeaked in our spectra. This means that at least the central part of the line probably originates from a region with a lower Keplerian velocity component than the one of the second ring of the [Ca II] line, which still displays a double-peaked profile. A reasonable fit to this central line profile region was obtained for a deprojected rotational velocity of $17.5 \pm 0.5 \mathrm{~km} \mathrm{~s}^{-1}$ and a Gaussian of $6.0 \mathrm{~km} \mathrm{~s}^{-1}$ (left panel of Fig. 9). However, as in the case of the double-peaked profiles, the broad wings cannot be fit with such a single ring. Therefore, we searched again for the best combination of two rings. The resulting fit is shown in the right panel of Fig. 9 and the velocity parameters are included in Table 4

Our results suggest that the emission in the [Ca II] and the [O I] $\lambda 5577$ lines originate from the same two rings with Keplerian velocities of $38-39 \mathrm{~km} \mathrm{~s}^{-1}$ and $22 \mathrm{~km} \mathrm{~s}^{-1}$, respectively. In contrast, the first ring from which the [O I] $\lambda 6300$ line originates is located in between these two [Ca II] rings and coincides with the location of the CO-band head emitting ring, while the second is located much farther out (see also Sect 5.3 and Table 5).

For the sake of completeness, we would like to add that we also considered the case that the forbidden emission lines might originate from a continuous disk. For this scenario assumptions need to be made for the radial density distribution. A power law has proven to work reasonably well to reproduce the spectral energy distributions of pre-main-sequence stars' disks (see, e.g., Beckwith et al. 1990). The density distribution within a stellar wind or an equatorial outflow can also be approximated by a power law (with a different exponent, however, see Borges Fernandes et al. 2007; Kraus et al. 2010). However, none of the studied scenarios resulted in a reasonable fit to the observed line profiles because they either were able to fit the broad wings but then produced too broad profiles in the central line regions, or they were able to reproduce the central regions but failed in producing broad enough line wings. 


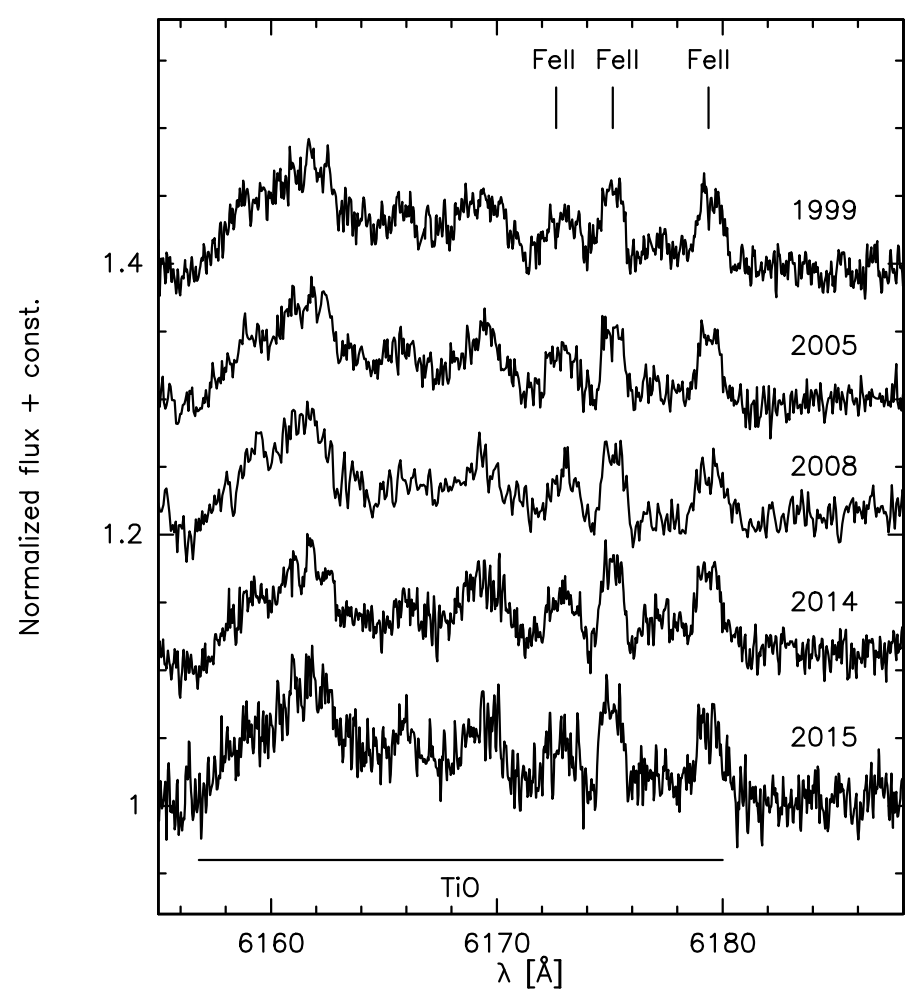

Fig. 10. Detection of TiO-band emission in the high-resolution optical spectra of LHA 120-S 73.

Considering that the double-peaked profiles have contributions from two distinct regions (closer to the star and farther out than the $\mathrm{CO}$ ring) that require similar densities, a continuous disk with a monotonic radial density distribution does not seem a very likely scenario for the distribution of the circumstellar material of $\mathrm{B}[\mathrm{e}] \mathrm{SG}$. Instead, our model results give preference to a scenario that involves multiple (sharp and/or extended in radial direction) density concentrations, which could either indicate a strongly non-monotonic radial density distribution within a continuous disk or just individual rings of material. Support for such a scenario comes from recent imaging result of one Galactic $\mathrm{B}$ [e]SG, for which multiple clumpy rings were resolved in the molecular CO gas (Kraus 2016) and also in the atomic gas (Kraus et al., in prep.).

\subsubsection{TiO-band emission}

The optical spectra display a broad emission feature extending from $\sim 6158 \AA$ to roughly $6180 \AA$ (see Fig. 10). At the faint red end, some Fe II lines are superimposed, but the blue part of the feature is not affected by emission lines. Similar structures were clearly seen in three other B[e]SGs, the two LMC stars LHA 120-S 12 and LHA 120-S 134, and the Small Magellanic Cloud (SMC) object LHA 115-S 18, and were assigned to TiO-band emission (Zickgraf et al. 1989; Torres et al. 2012). LHA 120-S 73 is hence the fourth object with clear signatures of TiO-band emission. Interestingly, all stars with $\mathrm{TiO}$ also display strong CO-band emission (see, e.g., Oksala et al. 2013). This suggests that the conditions in the environments (in terms of column density and emitting area) of these objects easily enable the production of measurable amounts of molecular emission. Hence emission from other species that form in oxygenrich environments, such as $\mathrm{SiO}$, which was recently discovered from four Galactic B[e]SGs (Kraus et al. 2015b), might also be detectable.

\section{Discussion}

\subsection{CO variability}

In Sect 4.1 we have shown that the observed variability in the CO-band emission can result from simultaneous variations in the CO column density and the size of the emitting area. On the other hand, no noticeable change in rotation velocity of the gas ring was observed. Such a behavior cannot be explained with a dilution of the emission that is due to expansion of the ring, for example, because in this case the rotation velocity would be affected, that is, it would be decreased. In addition, an increase in emitting area together with a decrease in column density would be expected. However, the model fits do not agree with such a scenario. The fit to the spectrum taken in December 2010 requires a $25 \%$ higher column density and simultaneously a $15 \%$ larger emitting area than is shown by the values obtained from fitting the data taken 11 days later (January 2011, see Table 3). Therefore, we interpret the observed variability in the CO intensity as due to density inhomogeneities within the molecular ring.

We stress that the variability in $\mathrm{CO}$ intensity cannot result from the motion of the gas ring during 11 days, even if this ring has density inhomogeneities. Considering the mass estimate of $30 M_{\odot}$ (Stahl et al. 1983) for the initial mass of LHA 120-S 73 and the Keplerian rotation velocity of $34 \mathrm{~km} \mathrm{~s}^{-1}$, the CO ring must have a radius of about $23 \mathrm{AU}$ and, hence, an orbital period of about $20 \mathrm{yr}$. Even if we assume that the current mass of LHA 120-S 73 is lower because the star experienced mass loss during its past evolution (of $\sim 10-30 \%$ of its initial mass), the orbital period would still exceed $10 \mathrm{yr}$. Therefore, 11 days are definitely too short for the motion of the gas to have a measurable influence on the line profiles. The observed $\mathrm{CO}$ variability must hence result from the intrinsic disposition of the inhomogeneities within the molecular ring.

To test this interpretation, we computed the line profiles of an optically thin gas ring with artificially implemented gaps. For simplicity and pure illustration purposes, we implemented two symmetric gaps into the ring, which otherwise remained unchanged (Keplerian rotation velocity of $34 \mathrm{~km} \mathrm{~s}^{-1}$, inclination angle of $28^{\circ}$ ). The symmetry was suggested by the three resolved rotation-vibrational lines in front of the second band head, which displayed no significant deviation from a symmetric doublepeaked profile shape. Depending on the location and size of the gaps in the ring, the individual line profiles change substantially. This is demonstrated in Fig. 11, where we compare the profiles of a line formed in a homogeneous ring (left panel) with those formed in rings with symmetric gaps positioned at the highest (middle panel) and lowest (right panel) velocities and an angular size of $20^{\circ}$ each. The effect on the line profiles is clearly evident: while the peak intensities are suppressed when the gaps are at the highest velocities, the central emission is strongly reduced in the model where the gaps are located at the lowest velocities.

If we recall that the structure of the CO-band heads results from the superposition of many individual rotation-vibrational lines (see, e.g., Carr 1995), it is obvious that the modifications and deformations of the individual profiles induced by the gaps considerably affect the shape and strength of the total CO-band emission. Hence, to explain the deviations between the model fit and the observations, the scenario of a clumpy structure of the molecular material seems to be most likely. We currently have no information on the number of clumps (or gaps), their sizes, 


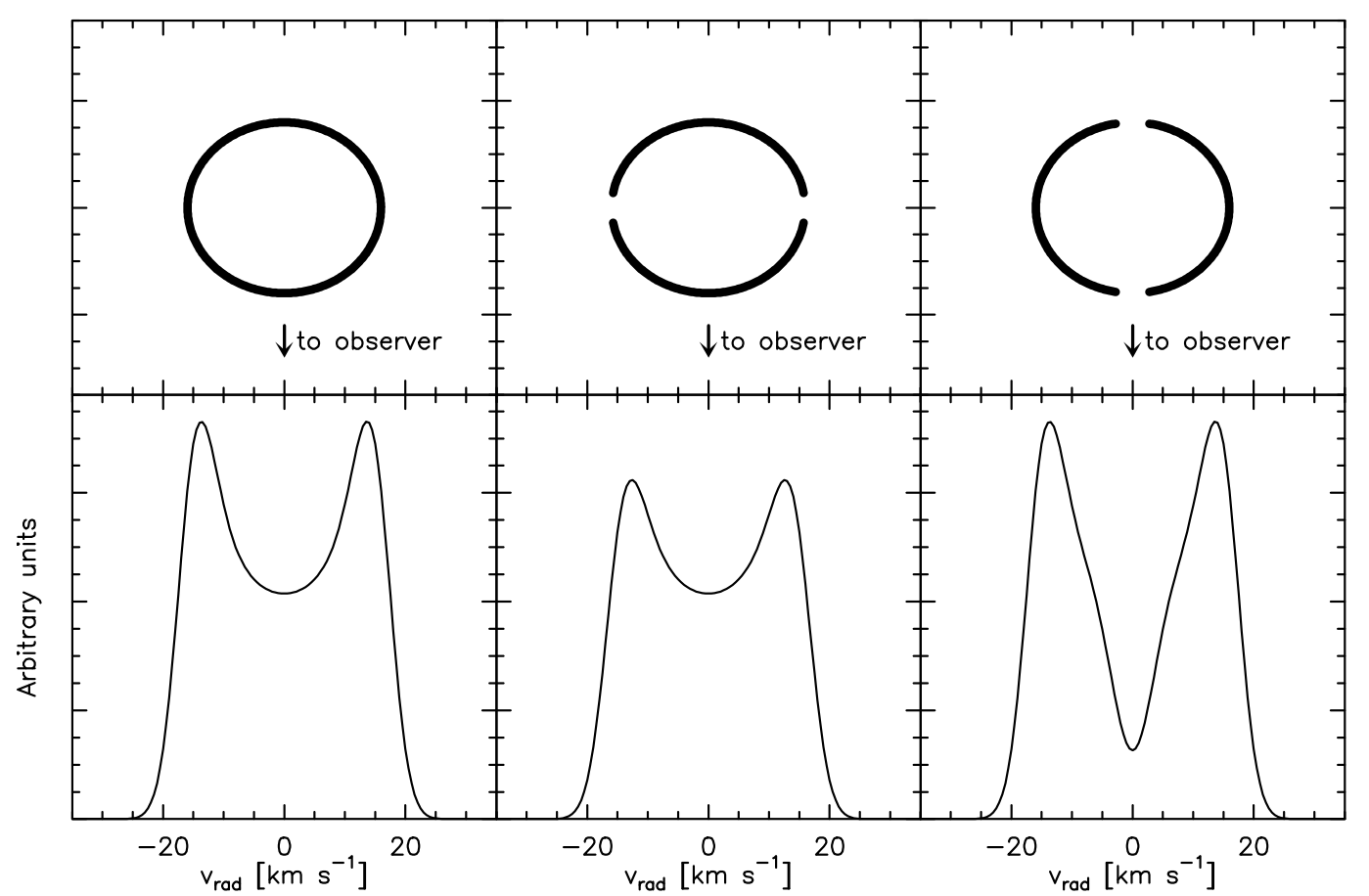

Fig. 11. Line profiles (bottom panels) resulting from different ring structures (top panels): homogeneous (left) or with symmetric gaps (middle and right).

surface densities, and positioning around the star at the time of observation. Such a clumpy ring structure has too many free parameters, hampering the proper modeling of the observed $\mathrm{CO}$ band spectra of LHA 120-S 73.

Further support that such a clumpy ring scenario could be reasonable is provided from a comparison of our results with those obtained recently by Oksala et al. (2013). These authors observed LHA 120-S 73 in 2009 as part of their $K$-band survey performed with the ESO medium-resolution $(R \sim 4500)$ integral-field unit spectrograph SINFONI. From their modeling of the CO-band emission, they derived a $\mathrm{CO}$ temperature of $2800 \pm 500 \mathrm{~K}$ that compares well to our value. However, the $\mathrm{CO}$ column density obtained by Oksala et al. (2013) is about a factor of six times higher than ours. We note that Oksala et al. (2013) modeled their spectrum without considering rotation of the $\mathrm{CO}$ gas because the structure of the CO-band head did not display noticeable kinematical broadening beyond the spectral resolution of SINFONI. While the difference in the treatment of the underlying kinematics certainly influences the shape and strength of the observable $\mathrm{CO}$ bands (as we discussed above), the deviation is too large to have a purely kinematic origin. Therefore, we suspect that the intensity of the SINFONI CO-band spectrum was different. To compare the intensities of the band head structures observed in different epochs, we convolved our highresolution Phoenix spectra taken in 2004 and 2010 to the resolution of the SINFONI spectrum of Oksala et al. (2013) taken in 2009. The result is shown in Fig. 12. When we compare the intensities of the first CO-band head emission, it is revealed that this has dropped from 2004 to 2009 and increased again in 2010 but remained below the intensity observed in 2004. This supports the idea that the molecular gas has a clumpy structure and revolves around the star on stable Keplerian orbits.

As was mentioned in Sect. 1, CO observations in B[e]SGs are rare, therefore not much is known about their possible variability. However, four other objects were reported with clear changes in the molecular emission. The Galactic object CI Cam

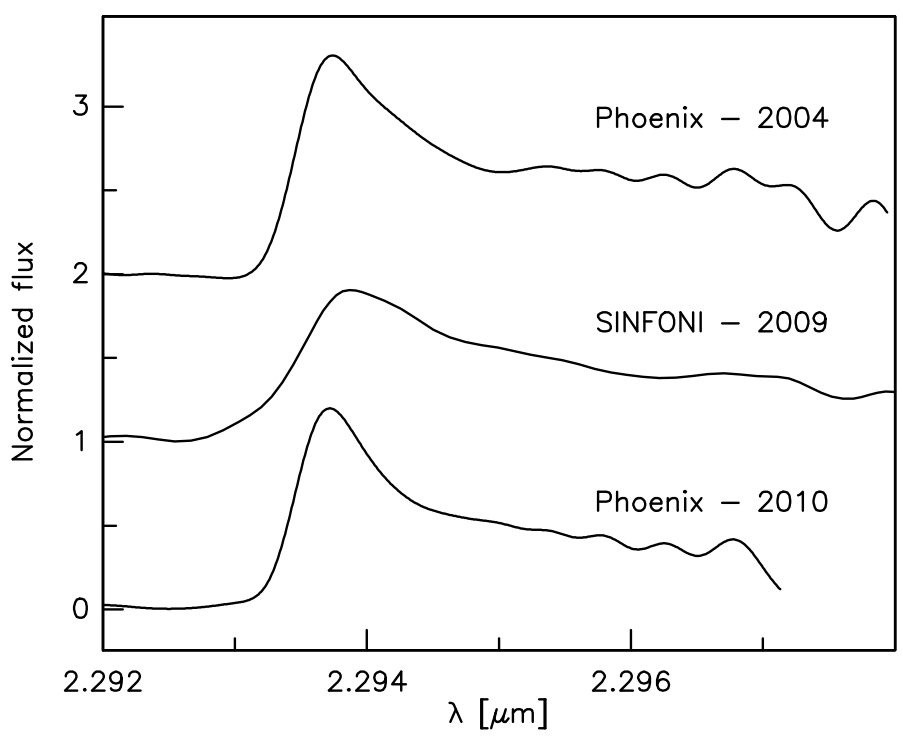

Fig. 12. Intensities of the first CO-band head observed in different years. For better comparison, the Phoenix spectra have been convolved to the resolution of the SINFONI spectrum of Oksala et al. (2013).

displayed strong CO-band emission in observations taken one month after its spectacular outburst in 1998 (Clark et al. 1999). This emission was no longer detectable in recent observations (Liermann et al. 2014). The B[e]SG star LHA 115-S 65 in the SMC displayed a very stable and long-lived disk with no CO bands seen in the past decades, while Oksala et al. (2012) reported on the sudden appearance of strong CO-band emission in that object. Oksala et al. (2013) detected CO-band emission in the B[e]SG star LHA 120-S 35 in the LMC, while the observations of Torres et al. (in prep.) taken approximately two years later revealed that the intensity of the CO-band emission in that object has decreased by about a factor of 
two. The Galactic B[e]SG star HD 327083 was reported to display CO-band emission by McGregor et al. (1988a). The CO bands were spatially resolved by Wheelwright et al. (2012b), who, based on interferometric observations combined with lowresolution $K$-band spectra, found no evidence for variability in $\mathrm{CO}$ within the observing period of five months. In contrast, high-resolution observations taken with a separation of only one month revealed noticeable differences in the shape of the CO-band head (Kraus et al. 2013a). Moreover, fully resolved rotation-vibrational lines in these data clearly display asymmetries in their double-peaked profiles: during the first observation the lines have a slightly stronger red peak, but they display a stronger blue peak one month later. While the observed variability in CI Cam and LHA 115-S 65 (and maybe also in LHA 120S 35) seems to be connected with highly dynamical phenomena (eruption, rapid expansion), HD 327083 could be another example of a B[e]SG with a stable, clumpy molecular ring.

\subsection{Origin of the circumstellar rings}

Combining the information obtained from the atomic and molecular gas tracers, it seems that LHA 120-S 73 is surrounded by multiple (at least four) gas rings. These rings populate distinct distances from the star, fixed by their Keplerian rotation speeds. Moreover, the fact that the first [OI] $\lambda 6300$ ring is situated in between the two [Ca II] rings indicates that the density in the individual rings does not decrease from inside out. Instead, the two [Ca II] rings must have clearly higher densities than the [O I] $\lambda 6300$ rings, otherwise we would expect contributions of these ring regions to the [Ca II] line profiles as well. In addition, the line profiles in our spectra taken over a period of $16 \mathrm{yr}$ display no measurable change in width. This means that the emitting regions must be stable in position. The only noticeable variability in the line profiles of the forbidden emission lines occur in the peak intensities (see Fig. 7). This could indicate that the density in these rings also displays some inhomogeneities.

The formation mechanism of rings and disks revolving the $\mathrm{B}$ [e]SGs on stable Keplerian orbits is still a major unsolved problem (see de Wit et al. 2014, for a recent review). The most commonly discussed scenarios are binary interaction up to full merger, and equatorially enhanced mass loss due to the rotationally induced bistability mechanism, which might be combined with a slow-wind solution. However, each of these scenarios has difficulties in reproducing all of the observed properties of $\mathrm{B}$ [e]SGs.

So far, indication for a companion was seen in only six B[e]SGs. Four of these stars (MWC 300, HD 327083, HD 62623, and GGCar) are Galactic objects, and in all of them the disk appears to be circumbinary (Wang et al. 2012; Millour et al. 2011; Wheelwright et al. 2012a; Kraus et al. 2013b), suggesting that binary interaction might have caused the formation of these disks. The other two objects, LHA 115-S 6 and LHA 115$\mathrm{S} 18$, reside in the SMC. LHA 115-S 6 was suggested to be a post-merger object in an original triple system (Langer \& Heger 1998; Podsiadlowski et al. 2006). This scenario was proposed because it explains why the less massive component appears more evolved than the more massive one. The other object, LHA 115-S 18, was identified as the optical counterpart of a high-mass X-ray binary source (Clark et al. 2013; Maravelias et al. 2014). Both SMC objects display variabilities and features in their spectra that clearly separate them from the other B[e]SGs in the Magellanic Clouds, of which none displays indication for a companion. We cannot exclude that these remaining objects are all merger remnants. However, in contrast to the strong variability and the diversity in spectral features observed in LHA 115-S 6, this scenario appears less likely.

The density enhancement in the equatorial plane achievable with the rotationally induced bistability mechanism is a factor 10-100 too low compared to the observations (Pelupessy et al. 2000). When combined with the slow-wind solution, the bistability mechanism is able to produce equatorial density enhancements of the right order (Curé et al. 2005). However, in that case, the wind velocities in the equatorial plane are vastly too high compared to the observed values. An additional hindrance with respect to LHA 120-S 73 is the relatively low stellar effective temperature of only $12000 \mathrm{~K}$, while the bistability jump occurs at temperatures around $22000 \mathrm{~K}$. A second bistability jump was suggested to occur around $10000 \mathrm{~K}$ (see Petrov et al. 2016), suitable to enhance the mass-loss rate in metal-rich objects close to the Eddington limit. However, as a member of the LMC and with an initial mass of only $\sim 30 M_{\odot}$, LHA $120-S 73$ does not necessarily fit into this group. Nevertheless, Curé et al. (2011) found that in this low-temperature regime a slower and denser wind exists that fits the observed terminal velocities and the windmomentum luminosity relation of A-type supergiants. Whether this new so-called delta-slow wind solution might also be relevant in the slightly hotter late $\mathrm{B}[\mathrm{e}]$ supergiant stars still needs to be tested.

Moreover, a prerequisite for the bistability mechanism is that the star should rotate at a substantial fraction of its critical velocity. LHA $120-S 73$ is one of only four B[e]SGs for which the rotation velocity projected to the line of sight could be derived. Zickgraf (2006) estimated a value of $v \sin i \simeq 50 \mathrm{~km} \mathrm{~s}^{-1}$ and a critical velocity of $\sim 140 \mathrm{~km} \mathrm{~s}^{-1}$. Considering the inclination angle of $28^{\circ}$, the deprojected rotation velocity would be $\sim 106 \mathrm{~km} \mathrm{~s}^{-1}$ and hence $\sim 75 \%$ of the critical velocity. This value is similar to what was previously found for two B[e]SGs in the SMC, LHA 115-S 65 (Zickgraf 2000; Kraus et al. 2010) and LHA 115-S 23 (Kraus et al. 2008), and would be sufficiently high for the bistability mechanism. However, we question the accuracy of the value of $v \sin i \simeq 50 \mathrm{~km} \mathrm{~s}^{-1}$.

For the measurement of $v$ sin $i$ Zickgraf (2006) used the He I $\lambda 5876$ line, as it is the only obvious, pure atmospheric line seen in the spectrum. A few other lines are present as well, but they are too shallow and hence very noisy. We computed an artificial line profile, considering only rotational broadening with $v \sin i=50 \mathrm{~km} \mathrm{~s}^{-1}$, thermal broadening for an effective temperature of $12000 \mathrm{~K}$, and broadening by the convolution with the spectral resolution. The result is shown in Fig. 13, where we overplot the artificial profile on those of the He I $\lambda 5876$ line observed in the different epochs. Two obvious conclusions can be drawn from this comparison: (i) the observed line profiles look not as roundish as expected if they would originate from predominantly stellar rotational broadening, in contrast to the theoretical line profiles; and (ii) the He I $\lambda 5876$ line is variable in width and strength and quite asymmetric.

This latter property is of particular interest. While we cannot exclude that the absorption line is polluted with emission from the wind and the circumstellar environment, the asymmetries strongly recall features visible in the profiles of non-radially pulsating stars (see, e.g., Telting \& Schrijvers 1997; Aerts et al. 2010a). Pulsations in LHA 120-S 73 were previously suggested by van Genderen \& Sterken (2002), who found indication for two periodic variabilities of $\sim 380 \mathrm{~d}$ and $\sim 55.5 \mathrm{~d}$ in the photometric light curve, and by Szczygieł et al. (2010), who observed a period of 224 days in the ASAS $V$-band light curve. Stellar pulsations can strongly influence the width and shape of atmospheric line profiles. Consequently, the width of a line provides 


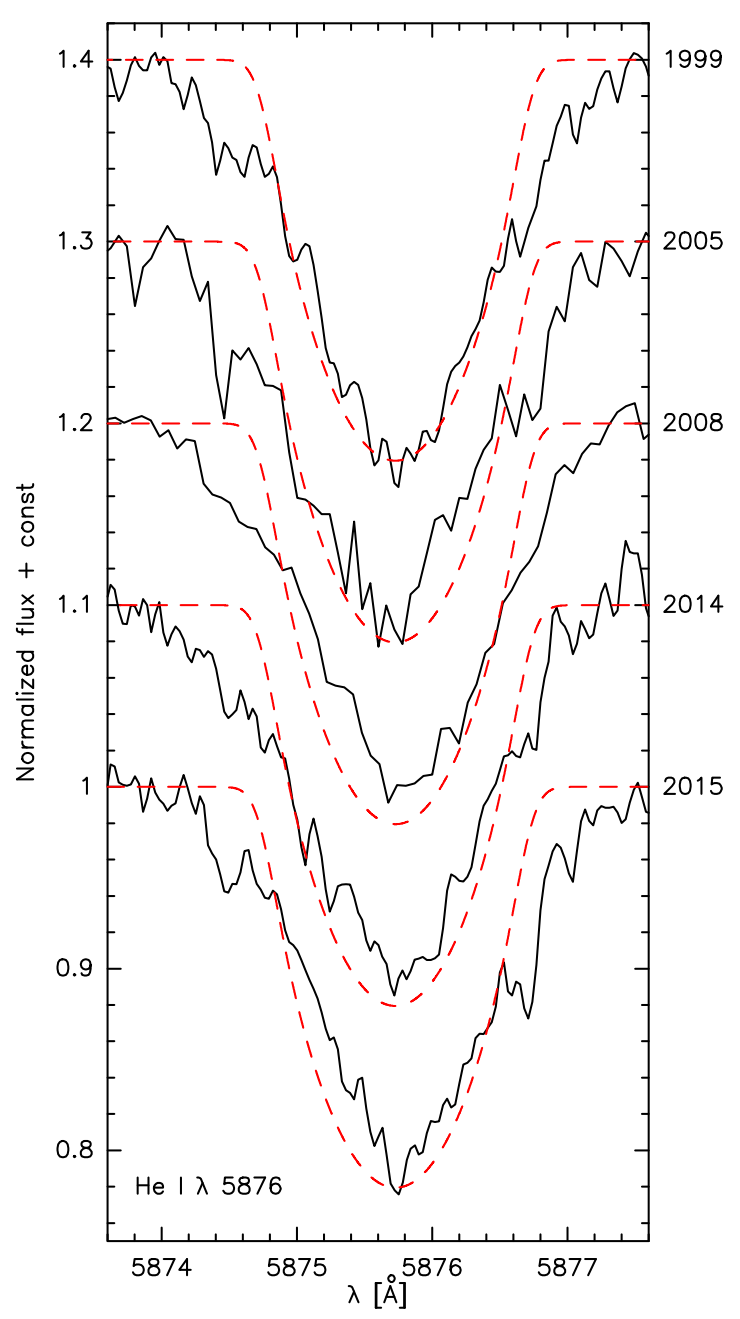

Fig. 13. Variability in the He I $\lambda 5876$ line.

no reliable indicator for the contribution of stellar rotation, and we suspect that $v \sin i$ of LHA 120-S 73 is (much) smaller than $50 \mathrm{~km} \mathrm{~s}^{-1}$. If present, pulsations might also (quasi-)periodically enhance the mass loss of the star (see, e.g., Aerts et al. 2010b; Kraus et al. 2015a), so that during specific times density peaks might form in the radial outflow. Depending on the pulsation modes, this "pulsed" mass loss might be confined within the equatorial plane, leading to ring structures in the outflow.

On the other hand, these rings seem to be quasi-stationary and recall ripple structures because no noticeable expansion of the emitting material is seen in our spectra over the observing period of $16 \mathrm{yr}$. Moreover, the sharpness of the observed line profiles, which indicates that the emission is confined to rather narrow rings, might imply that the space in between is mostly evacuated. This scenario strongly recalls ring systems around planets, in which moons have cleared the material in between the rings and stabilize the ring systems (so-called shepherd moons). Gaps populated by planets were also found in the accretion disks around pre-main-sequence objects (see, e.g., Rapson et al. 2015).

Although so far no planets were detected around evolved massive stars, the existence of one or more minor bodies revolving around LHA 120-S 73 and stabilizing the observed ring structures in analogy to the shepherd moons in planetary systems might offer a logical explanation. Observations of LHA 120-S 73 with the Spitzer Space Telescope IRS revealed an intense midinfrared excess emission with clear indications of amorphous
Table 5. Ring distances for $M_{*}=27 M_{\odot}$.

\begin{tabular}{llcc}
\hline \hline Ring No. & Element(s) & $\begin{array}{c}v_{\text {rot }} \\
\left(\mathrm{km} \mathrm{s}^{-1}\right)\end{array}$ & $\begin{array}{c}r \\
(\mathrm{AU})\end{array}$ \\
\hline 1 & {$[\mathrm{Ca} \mathrm{II}]([\mathrm{O} \mathrm{I}] \lambda$ 5577) } & 39 & 15.7 \\
2 & {$[\mathrm{O} \mathrm{I}] \lambda 6300, \mathrm{CO}$} & 34 & 20.7 \\
3 & {$[\mathrm{Ca}$ II] $([\mathrm{O} \mathrm{I}] \lambda 5577)$} & 22 & 49.5 \\
4 & {$[\mathrm{OI}] \lambda 6300$} & 16 & 93.6 \\
\hline
\end{tabular}

silicate dust. Moreover, specific spectral features originating from PAHs as well as from crystalline silicates were identified (Kastner et al. 2006) and are also seen in our mid-infrared spectrum (see Fig. 5) taken about six years later. The coexistence of crystalline silicates and PAHs requires that grain processing in non-equilibrium chemistry has taken place in a long-lived, stable dust disk. It remains unclear whether this dust disk might have provided the material for the formation of possible planets (or minor bodies) or whether such objects might have formed simultaneously with the star and survived the previous evolution.

\subsection{Evolutionary state of LHA 120-S 73}

The evolutionary state of B[e]SGs has long been unclear. The presence of large amounts of ejected material was often considered as a strong indication for a post-red supergiant (postRSG) stage. However, the investigations of Oksala et al. (2013) revealed that the chemical composition of the ejecta, in particular the enrichment in ${ }^{13} \mathrm{C}$, better agrees with a pre-RSG scenario. This is also the case for LHA 120-S 73, although it shows the strongest enrichment in ${ }^{13} \mathrm{C}$.

Additional support for a less evolved state is provided by the spectral energy distribution of the dust, which is clearly distinct from those of (post-)RSGs (see, e.g., Buchanan et al. 2006). Furthermore, LHA 120-S 73 is a point source in Spitzer IRAC/MIPS images without any diffuse nebulosity (Kastner et al. 2010).

Moreover, so far, only two photometric periodicities were found that might be interpreted as pulsational activity. Computations of Saio et al. (2013) for stars with an initial mass of $\sim 25 M_{\odot}$ have shown that only few pulsations with periods of weeks to months are expected in the effective temperature range of LHA 120-S 73, as long as the star is in the pre-RSG evolution. If LHA 120-S 73 were a post-RSG, it would undergo many more pulsations (Saio et al. 2013; Georgy et al. 2014). It is unclear as yet whether LHA 120-S 73 is indeed pulsating in just two modes. Nevertheless, based on the listed arguments, we may conclude that the star is most likely in a pre-RSG evolutionary state. Considering an initial mass of the object of $30 M_{\odot}$ and evolutionary tracks for rotating stars at LMC metallicity (Meynet \& Maeder 2005), the current mass of LHA 120-S 73 in a pre-RSG state would be about $27 M_{\odot}$. With this mass, we can compute the distances of the individual gas rings. The results are listed in Table 5.

\section{Conclusions}

We investigated the structure and kinematics of the circumstellar environment of the B[e]SG star LHA 120-S 73 in the LMC based on combined sets of high-resolution optical and near-infrared spectroscopic data collected over a time interval of 16 and $7 \mathrm{yr}$, respectively.

The near-infrared spectra cover the region of the first and second CO-band heads. The high spectral resolution revealed 
rotational broadening of the individual rotation-vibrational $\mathrm{CO}$ lines with a deprojected rotation velocity of $34 \mathrm{~km} \mathrm{~s}^{-1}$. In addition, we found that the CO-band head emission displays intensity variations, which we interpreted as density inhomogeneities within the molecular ring.

In the optical spectra we discovered an emission feature that we identified as molecular emission from TiO. LHA 120-S 73 is hence the fourth $\mathrm{B}[\mathrm{e}] \mathrm{SG}$ with confirmed TiO-band emission. In general, the optical emission features show no or only little indication for variability, suggesting that both the wind and the circumstellar material are in quasi-stationary state. The spectra display emission of the forbidden lines of [Ca II] and [O I], which are diagnostic tracers for circumstellar rings or disks. The lines have double-peaked profiles, which we interpreted, in analogy to previous studies, as due to Keplerian rotation. Modeling of the profile shapes revealed that each line profile consists of emission from two individual, spatially clearly distinct rings.

The optical spectra also reveal one clear absorption line, identified as He I $\lambda$ 5876. This line was formerly used to determine $v \sin i$ of the star and to demonstrate that the star is rapidly rotating. However, from our data we could not confirm the previously reported high value of $v \sin i$. Instead, our spectra show that this line is clearly variable and highly asymmetric, reminding of line profiles of non-radially pulsating stars. Although the $S / N$ of our data is too poor to favor a pulsational origin of the line asymmetries over line pollution due to emission, the presence of at least two periodic variabilities determined from the photometric light curve of LHA 120-S 73 provides evidence that pulsations might indeed play a non-negligible role in the dynamics of the stellar atmosphere.

In summary, we found that the global structure of the circumstellar material, traced by the forbidden emission lines and the $\mathrm{CO}$ molecular bands, consists of at least four distinct rings of alternating densities. Although we have currently no observational proof, we might speculate whether such a ripple structure of the circumstellar material might be caused by the presence of minor bodies (e.g. in the form of planets) that evacuated the space in between the rings, caused density inhomogeneities, and stabilize the currently observable ring structures, in analogy to the shepherd moons within the ring systems of planets or the rings observed around pre-main sequence objects. Follow-up observations with reasonable time resolution are clearly needed to investigate in more detail the variability, in particular of the molecular emission, to obtain better constraints on the density structure of the environment of LHA 120-S 73.

Acknowledgements. We thank the anonymous referee for comments that helped to improve the draft. This research made use of the NASA Astrophysics Data System (ADS) and of the SIMBAD database, operated at CDS, Strasbourg, France, and of Astropy, a community-developed core Python package for Astronomy (Astropy Collaboration et al. 2013). M.K., G.M., and D.H.N. acknowledge financial support from GA ČR (grant number 14-21373S). The Astronomical Institute Ondřejov is supported by the project RVO:67985815. This work was also supported by the institutional research fundings IUT26-2, IUT40-1, and IUT40-2 of the Estonian Ministry of Education and Research. L.C. acknowledges financial support from the Agencia de Promoción Científica y Tecnológica (Préstamo BID PICT 2011/0885), CONICET (PIP 0177), and the Universidad Nacional de La Plata (Programa de Incentivos G11/137), Argentina. Financial support for International Cooperation of the Czech Republic (MŠMT, 7AMB14AR017) and Argentina (Mincyt-Meys ARC/13/12 and CONICET 14/003) is acknowledged. M.C. acknowledges the support from FONDECYT project 1130173 and Centro de Astrofísica de Valparaíso, and R.H.B. acknowledges financial support from FONDECYT Project 1140076. The observations obtained in 2014 and 2015 with the MPG 2.2-m telescope were supported by the Ministry of Education, Youth and Sports project - LG14013 (Tycho Brahe: Supporting Ground-based Astronomical Observations). We would like to thank the observers (S. Ehlerova, A. Kawka) for obtaining the data.

\section{References}

Aerts, C., Christensen-Dalsgaard, J., \& Kurtz, D. W. 2010a, Asteroseismology (Springer Science Business Media B.V.)

Aerts, C., Lefever, K., Baglin, A., et al. 2010b, A\&A, 513, L11

Aret, A., Kraus, M., Muratore, M. F., \& Borges Fernandes, M. 2012, MNRAS, 423, 284

Aret, A., Kraus, M., \& Šlechta, M. 2016, MNRAS, 456, 1424

Astropy Collaboration, Robitaille, T. P., Tollerud, E. J., et al. 2013, A\&A, 558, A33

Beckwith, S. V. W., Sargent, A. I., Chini, R. S., \& Guesten, R. 1990, AJ, 99, 924 Bonanos, A. Z., Massa, D. L., Sewilo, M., et al. 2009, AJ, 138, 1003

Bonanos, A. Z., Lennon, D. J., Köhlinger, F., et al. 2010, AJ, 140, 416

Borges Fernandes, M., Kraus, M., Lorenz Martins, S., \& de Araújo, F. X. 2007, MNRAS, 377, 1343

Buchanan, C. L., Kastner, J. H., Forrest, W. J., et al. 2006, AJ, 132, 1890

Carr, J. S. 1995, Ap\&SS, 224, 25

Carr, J. S., Tokunaga, A. T., Najita, J., Shu, F. H., \& Glassgold, A. E. 1993, ApJ, 411, L37

Cidale, L. S., Borges Fernandes, M., Andruchow, I., et al. 2012, A\&A, 548, A72

Clark, J. S., Steele, I. A., Fender, R. P., \& Coe, M. J. 1999, A\&A, 348, 888

Clark, J. S., Bartlett, E. S., Coe, M. J., et al. 2013, A\&A, 560, A10

Curé, M., Rial, D. F., \& Cidale, L. 2005, A\&A, 437, 929

Curé, M., Cidale, L., \& Granada, A. 2011, ApJ, 737, 18

de Wit, W. J., Oudmaijer, R. D., \& Vink, J. S. 2014, Advances in Astronomy, 2014, 10

Domiciano de Souza, A., Bendjoya, P., Niccolini, G., et al. 2011, A\&A, 525, A22

Domiciano de Souza, A., Driebe, T., Chesneau, O., et al. 2007, A\&A, 464, 81

Ercolano, B., \& Owen, J. 2016, MNRAS, 460, 3472

Feast, M. W., Thackeray, A. D., \& Wesselink, A. J. 1960, MNRAS, 121, 337

Fehrenbach, C., Maurice, E., Prévot, L., \& Petit, M. 1969, A\&A, 3, 323

Georgy, C., Saio, H., \& Meynet, G. 2014, MNRAS, 439, L6

Henize, K. G. 1956, ApJS, 2, 315

Jones, O. C., Meixner, M., Sargent, B. A., et al. 2015, ApJ, 811, 145

Kastner, J. H., Buchanan, C. L., Sargent, B., \& Forrest, W. J. 2006, ApJ, 638, L29

Kastner, J. H., Buchanan, C., Sahai, R., Forrest, W. J., \& Sargent, B. A. 2010, AJ, 139, 1993

Kaufer, A., Stahl, O., Tubbesing, S., et al. 1999, The Messenger, 95, 8

Kraus, M. 2000, Ph.D. Thesis, Univ. Bonn, Germany

Kraus, M. 2009, A\&A, 494, 253

Kraus, M. 2016, Boletin de la Asociacion Argentina de Astronomia La Plata Argentina, 58, in press

Kraus, M., Krügel, E., Thum, C., \& Geballe, T. R. 2000, A\&A, 362, 158

Kraus, M., Borges Fernandes, M., \& de Araújo, F. X. 2007, A\&A, 463, 627

Kraus, M., Borges Fernandes, M., Kubát, J., \& de Araújo, F. X. 2008, A\&A, 487, 697

Kraus, M., Borges Fernandes, M., \& de Araújo, F. X. 2010, A\&A, 517, A30

Kraus, M., Cidale, L. S., Arias, M. L., et al. 2013a, in Massive Stars: From alpha to Omega, 160

Kraus, M., Oksala, M. E., Nickeler, D. H., et al. 2013b, A\&A, 549, A28

Kraus, M., Cidale, L. S., Arias, M. L., Oksala, M. E., \& Borges Fernandes, M. 2014, ApJ, 780, L10

Kraus, M., Haucke, M., Cidale, L. S., et al. 2015a, A\&A, 581, A75

Kraus, M., Oksala, M. E., Cidale, L. S., et al. 2015b, ApJ, 800, L20

Lamers, H. J. G. L. M., Zickgraf, F.-J., de Winter, D., Houziaux, L., \& Zorec, J. 1998, A\&A, 340, 117

Langer, N., \& Heger, A. 1998, in Proc. of the Workshop on B[e] stars, eds. A. M. Hubert, \& C. Jaschek (Kluwer Publ.), Astrophys. Space Sci. Libr., 233, 235

Liermann, A., Kraus, M., Schnurr, O., \& Fernandes, M. B. 2010, MNRAS, 408 L6

Liermann, A., Schnurr, O., Kraus, M., et al. 2014, MNRAS, 443, 947

Magalhaes, A. M. 1992, ApJ, 398, 286

Maravelias, G., Zezas, A., Antoniou, V., \& Hatzidimitriou, D. 2014, MNRAS, 438, 2005

McGregor, P. J., Hyland, A. R., \& Hillier, D. J. 1988a, ApJ, 324, 1071

McGregor, P. J., Hyland, A. R., \& Hillier, D. J. 1988b, ApJ, 334, 639

McGregor, P. J., Hyland, A. R., \& McGinn, M. T. 1989, A\&A, 223, 237

Meynet, G., \& Maeder, A. 2005, A\&A, 429, 581

Millour, F., Meilland, A., Chesneau, O., et al. 2011, A\&A, 526, A107

Morris, P. W., Eenens, P. R. J., Hanson, M. M., Conti, P. S., \& Blum, R. D. 1996 ApJ, 470, 597

Muratore, M. F., Kraus, M., Oksala, M. E., et al. 2015, AJ, 149, 13

Muratorio, G., \& Friedjung, M. 1988, A\&A, 190, 103

Najita, J., Carr, J. S., Glassgold, A. E., Shu, F. H., \& Tokunaga, A. T. 1996, ApJ, 462,919 
Oksala, M. E., Kraus, M., Arias, M. L., et al. 2012, MNRAS, 426, L56

Oksala, M. E., Kraus, M., Cidale, L. S., Muratore, M. F., \& Borges Fernandes, M. 2013, A\&A, 558, A17

Pelupessy, I., Lamers, H. J. G. L. M., \& Vink, J. S. 2000, A\&A, 359, 695

Petrov, B., Vink, J. S., \& Gräfener, G. 2016, MNRAS, 458, 1999

Podsiadlowski, P., Morris, T. S., \& Ivanova, N. 2006, in Stars with the B[e] Phenomenon, eds. M. Kraus, \& A. S. Miroshnichenko, ASP Conf. Ser., 355, 259

Rapson, V. A., Kastner, J. H., Millar-Blanchaer, M. A., \& Dong, R. 2015, ApJ, 815, L26

Saio, H., Georgy, C., \& Meynet, G. 2013, MNRAS, 433, 1246

Stahl, O., \& Wolf, B. 1986, A\&A, 158, 371

Stahl, O., Wolf, B., Zickgraf, F.-J., et al. 1983, A\&A, 120, 287

Stahl, O., Wolf, B., Leitherer, C., \& de Groot, M. 1985, A\&AS, 61, 237

Szczygieł, D. M., Stanek, K. Z., Bonanos, A. Z., et al. 2010, AJ, 140, 14

Telting, J. H., \& Schrijvers, C. 1997, A\&A, 317, 723

Torres, A. F., Kraus, M., Cidale, L. S., et al. 2012, MNRAS, 427, L80

van Boekel, R., Min, M., Waters, L. B. F. M., et al. 2005, A\&A, 437, 189 van Genderen, A. M., \& Sterken, C. 2002, A\&A, 386, 926

van Genderen, A. M., Groot, M., \& The, P. S. 1983, A\&A, 117, 53

van Loon, J. T., Oliveira, J. M., Gordon, K. D., et al. 2010, AJ, 139, 68

Wang, Y., Weigelt, G., Kreplin, A., et al. 2012, A\&A, 545, L10

Wheelwright, H. E., de Wit, W. J., Oudmaijer, R. D., \& Vink, J. S. 2012a, A\&A, 538, A6

Wheelwright, H. E., de Wit, W. J., Weigelt, G., Oudmaijer, R. D., \& Ilee, J. D. 2012b, A\&A, 543, A77

Zickgraf, F. 2000, in IAU Colloq. 175: The B[e] Phenomenon in Early-Type Stars, eds. M. A. Smith, H. F. Henrichs, \& J. Fabregat, ASP Conf. Ser., 214, 26

Zickgraf, F.-J. 2006, in Stars with the B[e] Phenomenon, eds. M. Kraus, \& A. S. Miroshnichenko, ASP Conf. Ser., 355, 135

Zickgraf, F.-J., Wolf, B., Stahl, O., Leitherer, C., \& Klare, G. 1985, A\&A, 143, 421

Zickgraf, F.-J., Wolf, B., Leitherer, C., Appenzeller, I., \& Stahl, O. 1986, A\&A, 163,119

Zickgraf, F.-J., Wolf, B., Stahl, O., \& Humphreys, R. M. 1989, A\&A, 220, 206 